\title{
The association of smoking status with SARS-CoV-2 infection, hospitalisation and mortality from COVID-19: A living rapid evidence review (version 3)
}

\author{
David Simons ${ }^{1}$, Lion Shahab², Jamie Brown², Olga Perski \\ 1 Royal Veterinary College, RVC \\ 2 University College London, University of London
}

Funding: The author(s) received no specific funding for this work.

Potential competing interests: The author(s) declared that no potential competing interests exist.

\begin{abstract}
Background: SARS-CoV-2 is the causative agent of COVID-19, an emergent zoonotic disease which has reached pandemic levels and is designated a public health emergency of international concern. It is plausible that former or current smoking status are associated with infection, hospitalisation and/or mortality from COVID-19.
\end{abstract}

Objective: We aimed to estimate the association of smoking status with rates of i) infection, ii) hospitalisation, iii) disease severity, and iv) mortality from SARS-CoV2/COVID-19.

Methods: We adopted recommended practice for rapid evidence reviews, which involved limiting the search to main databases and having one reviewer extract data and another verify. Published articles and pre-prints were identified via Ovid MEDLINE, medRxiv and expertise within the review team. We included observational studies with community-dwelling or hospitalised adults aged $16+$ years who had been tested for SARS-CoV-2 infection or diagnosed with COVID-19, providing that data on smoking status were reported. The National Institutes of Health's Quality Assessment Tool for Observational Cohort and Cross-Sectional Studies was used to divide studies into 'good', 'fair' and 'poor' quality to address objectives of this review. Studies were judged as 'good' quality if they: i) had low levels of missing data on smoking status, ii) used a reliable self-report measure that distinguished between current, former and never smoking status, iii) used biochemical verification of smoking status and iv) adjusted 
analyses for potential confounding variables.

Results: Sixty-seven studies were included, 30 of which were conducted in China, 12 in the US, six in the UK, four in France, three in Mexico, three in Spain, two across multiple international sites, two in Italy, and one each from Iran, Israel, Korea, Kuwait and Switzerland. Eleven studies did not state the source for information on smoking status. Fifty-one studies reported current and/or former smoking status but had high levels of missing data and/or did not explicitly state whether the remaining participants were never smokers. Notwithstanding recording uncertainties, compared with national prevalence estimates, recorded current and former smoking rates in most studies were lower than expected. In six 'fair' quality studies, no significant difference was observed between current and never $(\mathrm{RR}=0.78,95 \% \mathrm{Cl}=0.55-1.11, \mathrm{p}=.17, \mathrm{I} 2=92 \%)$ or former and never smokers $(R R=1.07,95 \% \mathrm{Cl}=0.95-1.20, p=.24,12=61 \%)$ in the risk of testing positive for SARS-CoV-2. In five 'fair' quality studies, there was no significant difference between current and never $(\mathrm{RR}=1.12,95 \% \mathrm{Cl}=0.74-1.69, \mathrm{p}=$ $.48,12=84 \%)$ or former and never smokers $(R R=1.21,95 \% \mathrm{Cl}=0.82-1.79, p=.24,12$ $=81 \%$ ) in the risk of requiring admission to hospital following diagnosis of COVID-19. In three 'fair' quality studies, current smokers were at increased risk of greater disease severity compared with never smokers $(R R=1.37,95 \% \mathrm{Cl}=1.07-1.75, p=.01,12=$ $0 \%)$. No significant difference was observed between former and never smokers (RR = $1.51,95 \% \mathrm{Cl}=0.82-2.80, \mathrm{p}=.19,12=81 \%)$. In three 'fair' quality studies, there were inconsistent results on mortality from COVID-19 in current and former compared with never smokers.

Conclusions: Across 67 observational studies, there is substantial uncertainty about the associations between smoking and COVID-19 outcomes. The recorded smoking prevalence in hospitalised patients was lower than national estimates but this observation is inconsistent with there being no evidence of increased admission to hospital from five 'fair' quality studies of people who tested positive. There was limited evidence from 'fair' quality studies that current compared with never smoking is associated with greater disease severity in those hospitalised for COVID-19.

Implications: Unrelated to COVID-19, smokers are at a greater risk of a range of serious health problems, requiring them to be admitted to hospital. Given uncertainty around the association of smoking with COVID-19, smoking cessation remains a public health priority and high-quality smoking cessation advice including recommendations to use alternative nicotine should form part of public health efforts during this 
pandemic.

\section{Introduction}

COVID-19 is a respiratory disease caused by the emerging SARS-CoV-2 virus. Large age and gender differences in case severity and mortality have been observed in the ong oing COVID-19 pandemic ${ }^{1}$; however, these differences are currently unexplained. SARS-CoV-2 enters epithelial cells through the ACE2 receptor $^{2}$. Some evidence suggests that gene expression and subsequent receptor levels are elevated in the airway and oral epithelium of current smokers ${ }^{3,4}$, thus putting smokers at higher risk of contracting SARS-CoV-2. Other studies, however, suggest that nicotine downregulates the ACE2 receptor ${ }^{5}$. These uncertainties notwithstanding, both former and current smoking is known to increase the

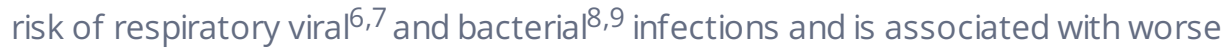
outcomes once infected. Cigarette smoke reduces the respiratory immune defence throug heri-bronchiolar inflammation and fibrosis, impaired mucociliary clearance and disruption of the respiratory epithelium ${ }^{10}$. There is also reason to believe that behavioural factors (e.g. regular hand-to-mouth movements) involved in smoking may increase SARS-CoV-2 infection and transmission in current smokers. However, early data from the COVID-19 pandemic have not provided clear evidence for a negative impact of current or former smoking on SARS-CoV-2 infection or COVID-19 disease outcomes, such as hospitalisation or mortality ${ }^{11}$. It has also been hypothesised that nicotine might protect against a hyper-inflammatory response (or "cytokine storm") to SARS-CoV-2 infection, which may lead to adverse outcomes in patients with COVID-19 disease ${ }^{12}$.

There are several reviews that fall within the scope of smoking and COVID-1911,13-17. We aimed to produce a rapid synthesis of available evidence pertaining to the rates of infection, hospitalisation, disease severity and mortality from SARS-CoV-2/COVID-19 stratified by smoking status. Given the increasing availability of data on this topic, this will be a 'living' review with fortnightly updates. As evidence accumulates, the review will be expanded to include studies reporting outcomes by alternative nicotine use (e.g., nicotine replacement therapy or e-cigarettes).

\section{Methods}

\section{Study design}

We adopted recommended practice for rapid evidence reviews, which involved limiting the search to main databases and having one reviewer extract the data and another 
verify ${ }^{18}$

Eligibility criteria

Studies were included if they:

1) Were primary research studies using experimental (e.g. randomised controlled trial), quasi-experimental (e.g. pre- and post-test) or observational (e.g. case-control) study designs;

2) Included adults aged $16+$ years;

3) Recorded as outcome i) results of a SARS-CoV-2 diagnostic test (including antibody assays), ii) a clinical diagnosis of COVID-19, iii) hospitalisation for COVID-19, iv) severity of COVID-19 disease or v) mortality from COVID-19;

4) Reported any of the outcomes of interest by self-reported or biochemically verified smoking status (e.g. current smoker, former smoker, never smoker);

5) Were available in English;

6) Were published in a peer-reviewed journal, as a pre-print or a public health report by reputable agents (e.g. governments, scientific societies).

\section{Search strategy}

The following terms were searched for in Ovid MEDLINE as free text or Medical Subject Headings:

1. Tobacco Smoking/ or Smoking Cessation/ or Water Pipe Smoking/ or Smoking/ or Smoking Pipes/ or Cigar Smoking/ or Smoking Prevention/ or Cigarette Smoking/ or smoking.mp. or Pipe Smoking/ or Smoking, Non-Tobacco Products/ or Smoking Water Pipes/

2. Nicotine/ or nicotine.mp. or Electronic Nicotine Delivery Systems/ or Nicotine Chewing Gum/

3. vaping.mp. or Vaping/

4. 1 or 2 or 3

5. Coronavirus/ or Severe Acute Respiratory Syndrome/ or Coronavirus Infections/ or covid.mp.

6. 4 and 5

The following terms were searched for in titles, abstracts and full texts in medRxiv:

1. covid smoking 
2. covid nicotine

3. covid vaping

Additional articles/reports of interest were identified through mailing lists, T witter, the International Severe Acute Respiratory and Emerging Infection Consortium (ISARIC), the Intensive Care National Audit \& Research Centre (ICNARC) and the US Centers for Disease Control and Prevention ( $\underline{C D C})$.

Where updated versions of pre-prints or reports were available, old versions were superseded.

\section{Selection of studies}

One reviewer screened titles, abstracts and full texts ag ainst the inclusion criteria.

\section{Data extraction}

Data were extracted by one reviewer and verified by a second on i) author (year); ii) date published; iii) country; iv) study design; v) study setting; vi) sample size; vii) sex; viii) age; ix) smoking status (e.g. current, former, never, missing); x) SARS-CoV-2 infection; xi) diagnosis of COVID-19; xii) hospitalisation for COVID-19; xiii) disease severity; and xiv) mortality.

\section{Quality appraisal}

The National Institutes of Health's Quality Assessment Tool for Observational Cohort and Cross-Sectional Studies was used to determine the quality (i.e. 'good', 'fair', 'poor') of included studies to address the specific objectives of our review ${ }^{19}$. In this context, studies were judged as 'good' quality if they: i) had low levels of missing data on smoking status, ii) used a reliable self-report measure that distinguished between current, former and never smoking status iii) used biochemical verification of smoking status; and iv) adjusted analyses for potential confounding variables (e.g. age, comorbidities). Studies were rated as 'fair' if they had low levels of missing data on smoking status and did one of either: i) used a reliable measure of current, former and never smoking status (e.g. self-report); or ii) adjusted analyses for potential confounding variables. Studies were otherwise rated as 'poor'. The quality appraisal was conducted by one reviewer and verified by a second.

\section{Evidence synthesis}

A narrative synthesis was conducted. Where possible, data were pooled in R v.3.6.3 ${ }^{20}$ with 
the Mantel-Haenszel or inverse variance method using random or fixed effects, depending on heterogeneity, and presented as risk ratios (RRs) ${ }^{21}$. Heterogeneity between study outcomes was assessed using the $\mathrm{I}^{2}$ statistic, suitable for smaller metaanalyses 22 .

\section{Results}

In the current review version (v3), a total of 143 new records were identified, with 67 studies included in a narrative synthesis and 12 studies included in meta-analyses (see Figure 1).

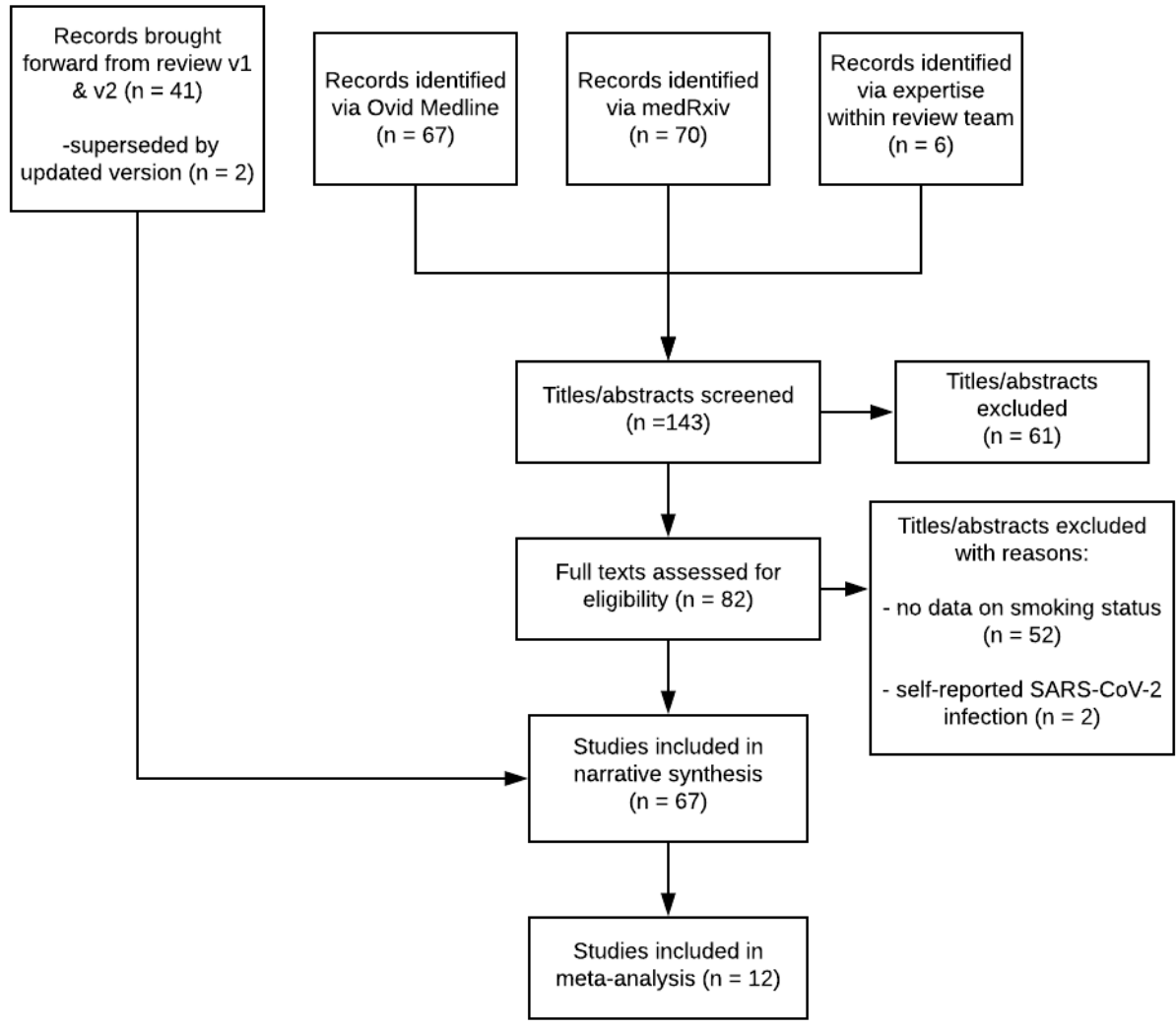

\section{Study characteristics}

Characteristics of included studies are presented in Table 1. Thirty studies were conducted in China ${ }^{1,23,32-41,24,42-51,25-31}$, twelve in the US $52,53,62,63,54-61$, six in the UK $^{64-69}$, four in France ${ }^{70-73}$, three in Mexico ${ }^{74-76}$, three in Spain ${ }^{77-79}$, two multi-site international studies 80,81 , two in Italy 82,83 , and with one each from Iran ${ }^{84}$, Israel ${ }^{85}$, Korea $^{86}$, Kuwait ${ }^{87}$ and Switzerland ${ }^{88}$. Fifty-four studies were conducted entirely in hospital settings. Thirteen studies included a community component in addition to 
hospitalised patients. Studies had a median of 393 (interquartile range $=101-1,402$ )

participants.

\section{Smoking status}

Categorisation of smoking status was heterogeneous (see T able 1). Eleven studies did not report the source for information on smoking status. Notably, only sixteen studies recorded current, former and never smoking status, with a further six studies reporting current or current/former and never smoking status. The remaining 45 studies reported current and/or former smoking status but did not explicitly state whether the remaining participants were never smokers or whether data on smoking status were missing. Nineteen studies explicitly reported missing data on smoking status, which ranged from $0.6 \%$ to $96 \%$. Smoking status was predominantly collected through routine electronic health records. Twenty studies used a bespoke case report form for COVID-19. None of the studies verified smoking status biochemically. Two studies ${ }^{26,41}$ specifically stated that smokers were those with a $>30$ pack-year history or a greater than 20-year history of smoking, respectively. Most studies did not assess tobacco exposure (e.g. pack-years of smoking) in current or former smokers, or time since quitting in former smokers. One study ${ }^{70}$ reported that $91.4 \%$ of former smokers had quit $\geq 6$ months prior to COVID-19 disease onset.

Table 1. Characteristics of included studies.

\begin{tabular}{|c|c|c|c|c|c|c|c|c|c|}
\hline Reference & Author & Date published & Country & Sample size & Setting & $\begin{array}{l}\begin{array}{l}\text { Median age } \\
\text { (IQR) }\end{array} \\
\end{array}$ & \% Female & Smoking status of those COVID+ & $\begin{array}{l}\text { Data source for smoking } \\
\text { status }\end{array}$ \\
\hline ["] & Guan, $\mathrm{Ni}$ & $28 / 02 / 2020$ & China & 1099 & Hospital & $47(35-58)$ & $41.9 \%$ & $\begin{array}{l}\text { Current smoker }(12.6 \%) \\
\text { Former smoker (1.9\%) } \\
\text { Never smoker }(85.4 \%)\end{array}$ & Not stated \\
\hline${ }^{[23]}$ & Guan, Liang & $26 / 03 / 2020$ & China & 1590 & Hospital & $49(33-64)$ & $42.7 \%$ & $\begin{array}{l}\text { Missing (1.3\%) } \\
\text { Current/former smoker }(7.0 \%)\end{array}$ & Not stated \\
\hline$[24]$ & Lian & $25 / 03 / 2020$ & China & 788 & Hospital & $\because$ & $38.5 \%$ & $\begin{array}{l}\text { Current smoker (6.9\%) } \\
\text { Not stated }(93.1 \%)\end{array}$ & Not stated \\
\hline$\left[{ }^{23]}\right]$ & $\operatorname{lin}$ & $24 / 03 / 2020$ & China & 651 & Hospital & $46(32-60)$ & $49.2 \%$ & $\begin{array}{l}\text { Current smoker (6.3\%) } \\
\text { Not stated (93.7\%) }\end{array}$ & Not stated \\
\hline$\left[{ }^{26}\right.$ ] & Chen & $26 / 03 / 2020$ & China & 548 & Hospital & $62(44-70)$ & $37.6 \%$ & $\begin{array}{l}\text { Current smoker }(4.4 \%)^{*} \\
\text { Former smoker }(2.6 \%)^{*} \\
\text { Not stated }(93.1 \%)\end{array}$ & Not stated \\
\hline$\left[{ }^{2}\right]$ & Zhou & $11 / 03 / 2020$ & China & 191 & Hospital & $56(46-67)$ & $38.0 \%$ & $\begin{array}{l}\text { Current smokerer (6.0\%) } \\
\text { Not stated (94.0\%) }\end{array}$ & Not stated \\
\hline$\left[{ }^{28}\right]$ & Mo & $16 / 03 / 2020$ & China & 155 & Hospital & $54(53-66)$ & $44.5 \%$ & $\begin{array}{l}\text { Current smoker (3.9\%) } \\
\text { Not stated (96.1\%) }\end{array}$ & Case report form \\
\hline$\left[{ }^{23}\right]$ & Zhang, Dong & $19 / 02 / 2020$ & China & 140 & Hospital & $57(25-87)^{\wedge}$ & $46.3 \%$ & $\begin{array}{l}\text { Current smoker }(1.4 \%) \\
\text { Former smoker }(5.0 \%) \\
\text { Not stated }(93.6 \%)\end{array}$ & Electronic health records \\
\hline$\left[{ }^{30}\right]$ & Wan & $21 / 03 / 2020$ & China & 135 & Hospital & $47(36-55)$ & $46.7 \%$ & $\begin{array}{l}\text { Current smoker (6.7\%) } \\
\text { Not stated (93.3\%) }\end{array}$ & Electronic health records \\
\hline$\left[^{33}\right]$ & Liu, Tao & $28 / 02 / 2020$ & China & 78 & Hospital & $38(33-57)$ & $50.0 \%$ & $\begin{array}{l}\text { Current/former smoker (6.4\%) } \\
\text { Not stated (93.6\%) }\end{array}$ & Case report form \\
\hline$\left[{ }^{32}\right]$ & Huang, Wang & $05 / 03 / 2020$ & China & 41 & Hospital & $49(41-58)$ & $27.0 \%$ & $\begin{array}{l}\text { Current smoker (7.3\%) } \\
\text { Not stated (92.7\%) }\end{array}$ & Electronic health records \\
\hline [3] & Zhang, Cai & $20 / 03 / 2020$ & China & 645 & Hospital & $\because$ & $49.1 \%$ & $\begin{array}{l}\text { Current smoker (6.4\%) } \\
\text { Not stated (93.5\%) }\end{array}$ & Electronic health records \\
\hline$\left[{ }^{34}\right]$ & Guo & $27 / 03 / 2020$ & China & 187 & Hospital & $59(45-73)$ & $51.3 \%$ & $\begin{array}{l}\text { Current smoker (9.6\%) } \\
\text { Not stated (90.4\%) }\end{array}$ & Electronic health records \\
\hline [33] & Liu, Ming & $12 / 03 / 2020$ & China & 41 & Hospital & $39(30-48)$ & $58.5 \%$ & $\begin{array}{l}\text { Current smoker (9.8\%) } \\
\text { Not stated }(90.2 \%)\end{array}$ & Electronic health records \\
\hline$\left[{ }^{32}\right]$ & Huang, Yang & $05 / 03 / 2020$ & China & 36 & Hospital & $69(60-78)$ & $30.6 \%$ & $\begin{array}{l}\text { Current/former smoker (11.1\%) } \\
\text { Not stated (88.9\%) }\end{array}$ & Not stated \\
\hline$\left[{ }^{33}\right]$ & $\mathrm{xu}$ & $08 / 03 / 2020$ & China & 53 & Hospital & $\because$ & $47.2 \%$ & $\begin{array}{l}\text { Current smoker (11.3\%) } \\
\text { Not stated (88.7\%) }\end{array}$ & Electronic health records \\
\hline$\left[{ }^{38}\right]$ & Li & $12 / 02 / 2020$ & China & 17 & Hospital & $45(33-57)$ & $47.1 \%$ & $\begin{array}{l}\text { Current smoker (17.6\%) } \\
\text { Not stated }(82.4 \%)\end{array}$ & Electronic health records \\
\hline
\end{tabular}




\begin{tabular}{|c|c|c|c|c|c|c|c|c|c|}
\hline$[22]$ & Rentsch $\sim$ & $14 / 04 / 2020$ & USA & 3789 & Community/hospital & $66(60-70)$ & $4.6 \%$ & $\begin{array}{l}\text { Current smoker }(27.2 \%) \\
\text { Former smoker }(30.6 \%) \\
\text { Never smoker }(36.9 \%)\end{array}$ & Electronic health records \\
\hline$\left[{ }^{39}\right]$ & $\mathrm{Hu}$ & $25 / 03 / 2020$ & China & 323 & Hospital & $61(23-91)^{\wedge}$ & $48.6 \%$ & $\begin{array}{l}\text { Missing (5.3\%) } \\
\text { Current/former smoker (11.8\%) }\end{array}$ & Not stated \\
\hline$\left[{ }^{40}\right]$ & Wang & $24 / 03 / 2020$ & China & 125 & Hospital & $41(26-66)$ & $43.2 \%$ & $\begin{array}{l}\text { Current/former smoker (11.8\%) } \\
\text { Not stated (87.2\%) }\end{array}$ & Electronic health records \\
\hline$[3]$ & Petrilli & $11 / 04 / 2020$ & USA & 4103 & Community/hospital & $52(36-65)$ & $47.9 \%$ & $\begin{array}{l}\text { Current smoker (5.2\%) } \\
\text { Former smoker (16.2\%) } \\
\text { Never smoker//unknown (78.6\%) }\end{array}$ & Electronic health records \\
\hline$\left[{ }^{3}\right]$ & Chow (US CDC) & $31 / 03 / 2020$ & USA & 7162 & Community/hospital & $\because$ & - & $\begin{array}{l}\text { Current smoker }(1.3 \%) \\
\text { Former smoker }(2.3 \%) \\
\text { Missing }(96.4 \%)\end{array}$ & Case report form \\
\hline$[\%]$ & Miyara & 09/05/2020 & France & 479 & Community/hospital & $\therefore$ & $44.7 \%$ & $\begin{array}{l}\text { Current/occasional smoker (6.7\%) } \\
\text { Former smoker (31.7\%) } \\
\text { Never smoker (59.7\%) } \\
\text { Missing (1.8\%) }\end{array}$ & Case report form \\
\hline$[4]$ & Dong & $20 / 03 / 2020$ & China & 9 & Hospital & $44(30-46)$ & $66.7 \%$ & $\begin{array}{l}\text { Current smoker (11.1\%)\# } \\
\text { Never smoker/unknown }(88.9 \%)\end{array}$ & Electronic health records \\
\hline$\left[{ }^{[8]}\right]$ & Kim & $01 / 04 / 2020$ & Korea & 28 & Hospital & $43(30-56)$ & $46.4 \%$ & $\begin{array}{l}\text { Current smoker }(18.5 \%) \\
\text { Never smoker/unknown }(81.5 \%)\end{array}$ & Electronic health records \\
\hline$\left[{ }^{42}\right]$ & Shi, Yu & $18 / 03 / 2020$ & China & 487 & Hospital & $46(27-65)$ & $46.8 \%$ & $\begin{array}{l}\text { Current/former smoker }(8.2 \%) \\
\text { Never smoker/unknown }(89.1 \%)\end{array}$ & Case report form \\
\hline$\left[{ }^{43}\right]$ & Yang, Yu & $24 / 02 / 2020$ & China & 52 & Hospital & $60(47-73)$ & $37.0 \%$ & $\begin{array}{l}\text { Current smoker (3.8\%) } \\
\text { Never smoker/unknown (96.2\%) }\end{array}$ & Case report form \\
\hline ["] & Argenziano & $22 / 04 / 2020$ & USA & 1000 & Hospital & $63(50-75)$ & $40.4 \%$ & $\begin{array}{l}\text { Current smoker }(4.9 \%) \\
\text { Former smoker }(17.9 \%) \\
\text { Never smoker }(77.2 \%)\end{array}$ & Case report form \\
\hline$\left[{ }^{74}\right]$ & Solis & $25 / 04 / 2020$ & Mexico & 650 & Hospital & $46^{\wedge}$ & $42.1 \%$ & $\begin{array}{l}\text { Current smoker (9.4\%) } \\
\text { Not stated (90.6\%) }\end{array}$ & Electronic health records \\
\hline$\left[{ }^{36}\right]$ & Richardson & $22 / 04 / 2020$ & USA & 5700 & Hospital & $63(52-75)$ & $39.7 \%$ & $\begin{array}{l}\text { Current/former smoker }(9.8 \%) \\
\text { Never smoker }(52.8 \%) \\
\text { Missing }(37.4 \%)\end{array}$ & Electronic health records \\
\hline$\left[{ }^{73}\right]$ & Fontanet & $23 / 04 / 2020$ & France & 661 & Community & $37(16-47)$ & $62.0 \%$ & $\begin{array}{l}\text { Current smoker (10.4\%) } \\
\text { Never smoker/unknown }(89.6 \%)\end{array}$ & Case report form \\
\hline$\left[{ }^{[4}\right]$ & Zheng, Gao & $19 / 04 / 2020$ & China & 66 & Hospital & $47^{\wedge}$ & $25.8 \%$ & $\begin{array}{l}\text { Current smoker }(12.1 \%) \\
\text { Not stated }(87.9 \%)\end{array}$ & Not stated \\
\hline$\left[{ }^{45}\right]$ & Liao, Feng & $24 / 04 / 2020$ & China & 1848 & Hospital & $55(48-61)$ & $54.7 \%$ & $\begin{array}{l}\text { Current/former smoker (0.4\%) } \\
\text { Not stated (7.6\%) } \\
\text { Missing (92.0\%) }\end{array}$ & Electronic health records \\
\hline$["]$ & Rodriguez-Cola & $24 / 04 / 2020$ & Spain & 7 & Hospital & $68(34-75)$ & $28.6 \%$ & $\begin{array}{l}\text { Current/former smoker (42.9\%) } \\
\text { Never smoker (57.1\%) }\end{array}$ & Electronic health records \\
\hline$\left[{ }^{27}\right]$ & Magagnoli & $16 / 04 / 2020$ & USA & 368 & Hospital & $69(59-75)$ & $0.0 \%$ & $\begin{array}{l}\text { Current/former smoker (14.1\%) } \\
\text { Not stated (85.9\%) }\end{array}$ & Electronic health records \\
\hline [4] ] & Shi, Ren & $23 / 04 / 2020$ & China & 134 & Hospital & $46(34-58)$ & $51.5 \%$ & $\begin{array}{l}\text { Current/former smoker (10.5\%) } \\
\text { Not stated }(89.5 \%)\end{array}$ & Case report form \\
\hline$\left[{ }^{72}\right]$ & Hadjadj & $23 / 04 / 2020$ & France & 50 & Hospital & $55(50-63)$ & $22.0 \%$ & $\begin{array}{l}\text { Current smoker }(2.0 \%) \\
\text { Former smoker (18.0\%) } \\
\text { Never smoker }(80.0 \%)\end{array}$ & Electronic health records \\
\hline [s] & Niedzwiedz & $30 / 04 / 2020$ & UK & 428,225 & Community and hospital & $\because$ & - & $\begin{array}{l}\text { Current smoker }(10.0 \%) \\
\text { Former smoker }(34.6 \%) \\
\text { Never smoker }(55.4 \%)\end{array}$ & Case report form \\
\hline ["8] & Gold (US CDC) & $20 / 04 / 2020$ & USA & 305 & Hospital & - & - & $\begin{array}{l}\text { Current smoker (5.1\%) } \\
\text { Not stated (94.9\%) }\end{array}$ & Case report form \\
\hline$\left[{ }^{80}\right]$ & Mehra & $01 / 05 / 2020$ & Multiple & 8910 & Hospital & $49^{\wedge}$ & $40.0 \%$ & $\begin{array}{l}\text { Current smoker }(5.5 \%) \\
\text { Former smoker (16.8\%) } \\
\text { Not stated }(77.7 \%)\end{array}$ & Case report form \\
\hline$\left[{ }^{81}\right]$ & ISARIC & $06 / 05 / 2020$ & Multiple & 20,276 & Hospital & $72(0-104)^{\wedge}$ & $40.0 \%$ & $\begin{array}{l}\text { Current/former smoker (4.8\%) } \\
\text { Never smoker (48.9\%) } \\
\text { Missing (46.3\%) }\end{array}$ & Case report form \\
\hline$\left[{ }^{47}\right]$ & Yu, Cai & $27 / 04 / 2020$ & China & 95 & Hospital & $\because$ & $44.2 \%$ & $\begin{array}{l}\text { Current smoker }(8.4 \%) \\
\text { Not stated }(91.6 \%)\end{array}$ & Electronic health records \\
\hline$\left[{ }^{49}\right]$ & Zheng, Xiong & $30 / 04 / 2020$ & China & 73 & Hospital & $43^{n}$ & $45.2 \%$ & $\begin{array}{l}\text { Current/former smoker }(11.0 \%) \\
\text { Never smoker }(89.0 \%)\end{array}$ & Not stated \\
\hline$\left[{ }^{79}\right]$ & de la Rica & $11 / 05 / 2020$ & Spain & 48 & Hospital & $66(33-88)^{\wedge}$ & $33 \%$ & $\begin{array}{l}\text { Current/former smoker (20.8\%) } \\
\text { Not stated (79.2\%) }\end{array}$ & Electronic health records \\
\hline$\left[{ }^{49}\right]$ & Yin, Yang & $10 / 05 / 2020$ & China & 106 & Hospital & $73(67-79)$ & $39.6 \%$ & $\begin{array}{l}\text { Current/former smoker (17.0\%) } \\
\text { Not stated }(83 \%)\end{array}$ & Electronic health records \\
\hline$\left[{ }^{22}\right]$ & Gaibazzi & $10 / 05 / 2020$ & Italy & 441 & Hospital & $71(62-80)$ & $38 \%$ & $\begin{array}{l}\text { Current smoker }(4.8 \%) \\
\text { Former smoker (10\%) } \\
\text { Never smoker }(85.3 \%)\end{array}$ & Case report form \\
\hline$[39]$ & Shi, Zuo & $10 / 05 / 2020$ & USA & 96 & Hospital & $63(54-74)^{\wedge}$ & $41 \%$ & $\begin{array}{l}\text { Current/former smoker }(30.2 \%) \\
\text { Not stated }(69.8 \%)\end{array}$ & Not stated \\
\hline [5] & Cho & $11 / 05 / 2020$ & UK & 1331 & Community and Hospital & $\because$ & $49.2 \%$ & $\begin{array}{l}\text { Current smoker (19\%) } \\
\text { Former smoker (27\%) } \\
\text { Never smoker (54\%) }\end{array}$ & Case report form \\
\hline$\left[{ }^{73}\right]$ & Allenbach & $08 / 05 / 2020$ & France & 152 & Hospital & $77(60-83)$ & $31.1 \%$ & $\begin{array}{l}\text { Current/former smoker (6.6\%) } \\
\text { Not stated (93.4\%) }\end{array}$ & Electronic health records \\
\hline$\left[{ }^{60}\right]$ & Robilotti & $08 / 05 / 2020$ & USA & 423 & Hospital & $\therefore$ & $50 \%$ & $\begin{array}{l}\text { Current smoker }(2.1 \%) \\
\text { Former smoker }(37.6 \%) \\
\text { Never smoker }(58.6 \%) \\
\text { Missing }(1.7 \%)\end{array}$ & Electronic health records \\
\hline [6] & $\begin{array}{l}\text { OpenSAFELY } \\
\text { Collaborative }\end{array}$ & 07/05/2020 & UK & $17,425,445$ & Community and Hospital & $\because$ & $50.1 \%$ & $\begin{array}{l}\text { Current smoker }(17.0 \%) \\
\text { Former smoker (32.9\%) } \\
\text { Never smoker }(45.9 \%) \\
\text { Missing (4.2\%) }\end{array}$ & Electronic health records \\
\hline$[79]$ & Borobia & 06/05/2020 & Spain & 2226 & Hospital & $61(46-78)$ & $52 \%$ & $\begin{array}{l}\text { Current smoker (7.1\%) } \\
\text { Not stated (92.9\%) }\end{array}$ & Case report form \\
\hline$\left[{ }^{33}\right]$ & Giacomelli & 06/05/2020 & Italy & 233 & Hospital & 50-72) & $31.9 \%$ & $\begin{array}{l}\text { Current/former smoker (30\%) } \\
\text { Never smoker (70\%) }\end{array}$ & Case report form \\
\hline
\end{tabular}




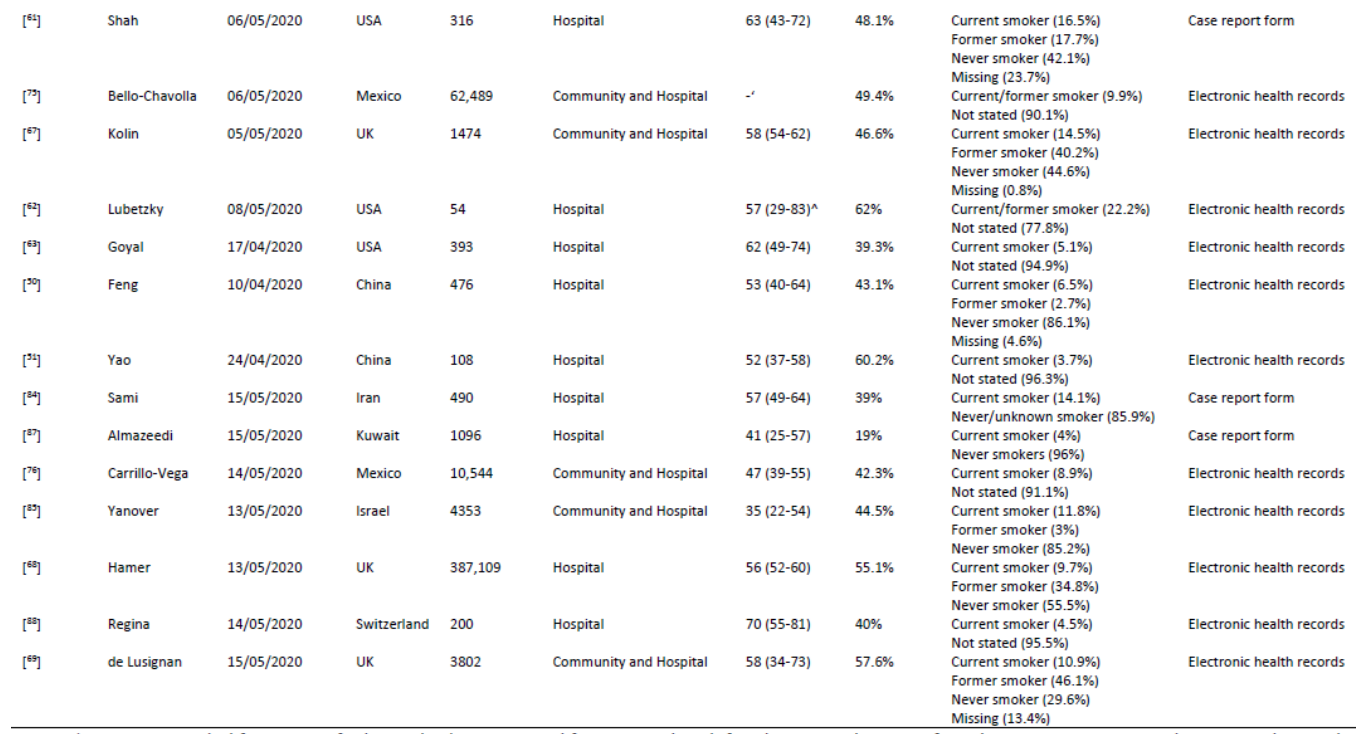

Note. -'Age not provided for unstratified sample; * Current and former smoker defined as 30 pack-years of smoking; ^ Denotes range (as opposed to IQR); $\sim$ Includes participants with negative and positive SARS-CoV-2 tests; \# Current smoker defined as >20 years of smoking.

\section{Smoking prevalence by country}

Observed smoking prevalence by country is presented in Figure 2. Overall, compared with national smoking prevalence, lower than expected current and former smoking rates were observed in most studies across all countries.

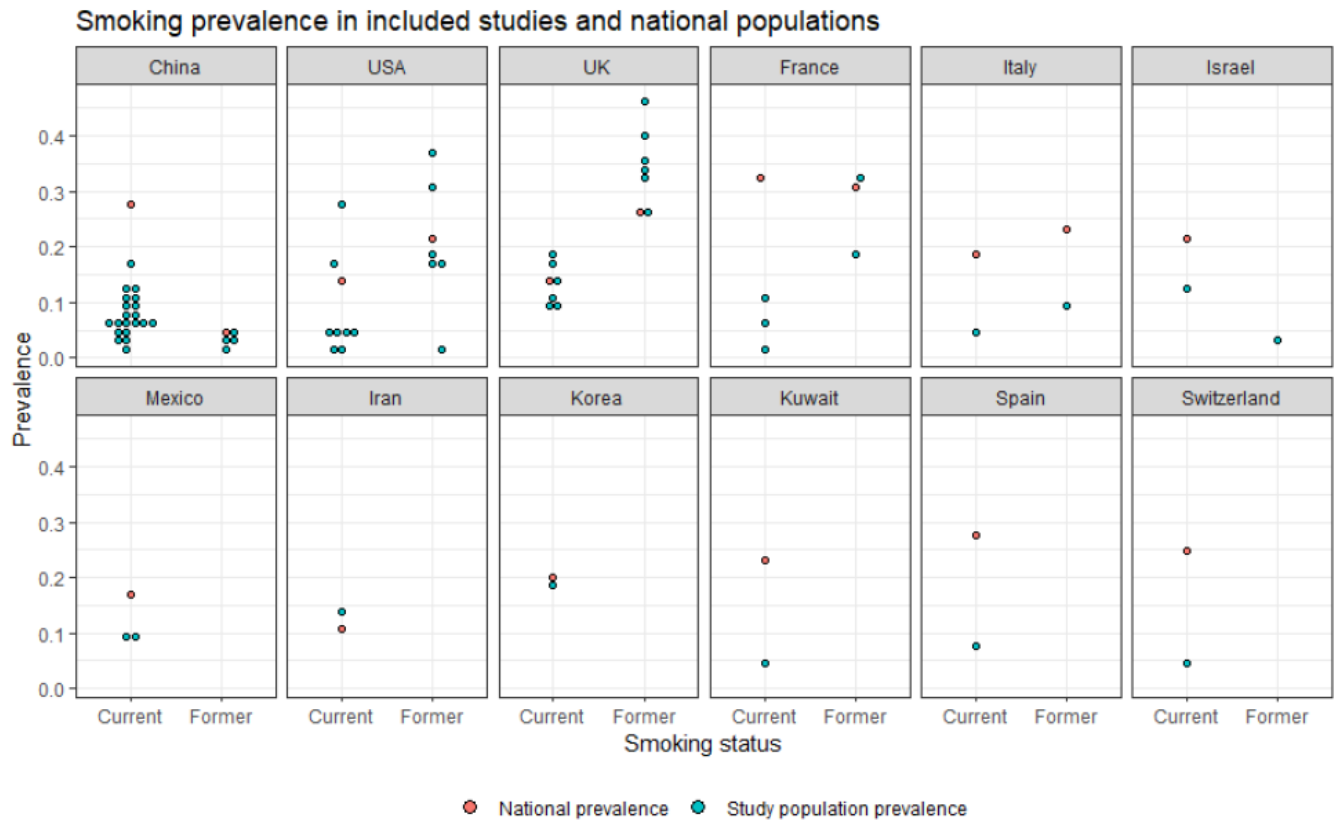

Figure 2. Observed compared with expected smoking prevalence by country. No national data on former smoking prevalence for Israel were identified. Studies in countries presented in the lower panel did not report former smoking prevalence. 


\section{SARS-COV-2 infection by smoking status}

Two 'poor' and six 'fair' quality studies provided data on SARS-CoV-2 test results for people meeting local testing criteria by smoking status (see Table 2). Meta-analyses were performed for the six 'fair' quality studies. No significant difference was observed between current and never smokers $(\mathrm{RR}=0.78,95 \% \mathrm{Cl}=0.55-1.11, \mathrm{p}=.17)$ or former and never smokers $(\mathrm{RR}=1.07,95 \% \mathrm{Cl}=0.95-1.20, \mathrm{p}=.24)$ in the risk of testing positive for SARS-CoV-2 (see Figure 3 and 4, respectively).

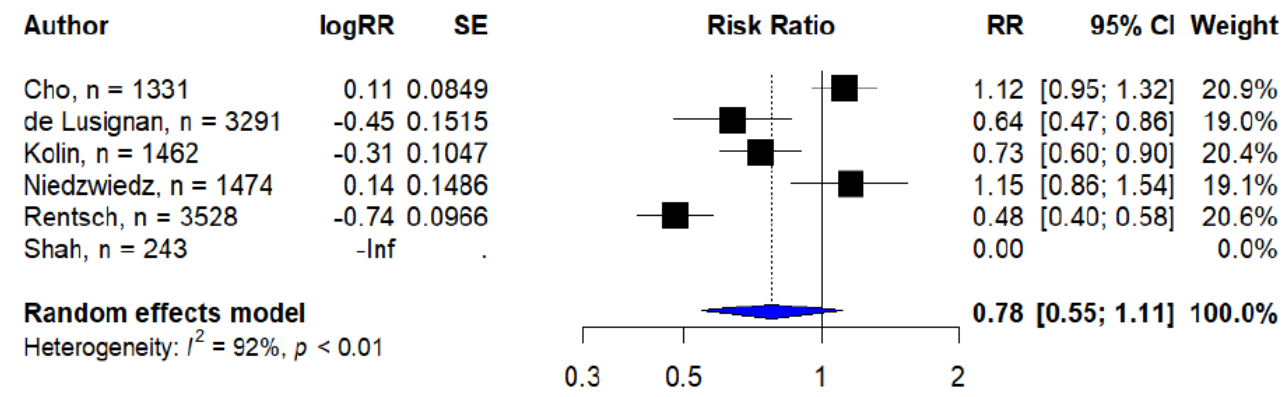

Figure 3. Forest plot for risk of testing positive for SARS-CoV-2 in current vs. never smokers.

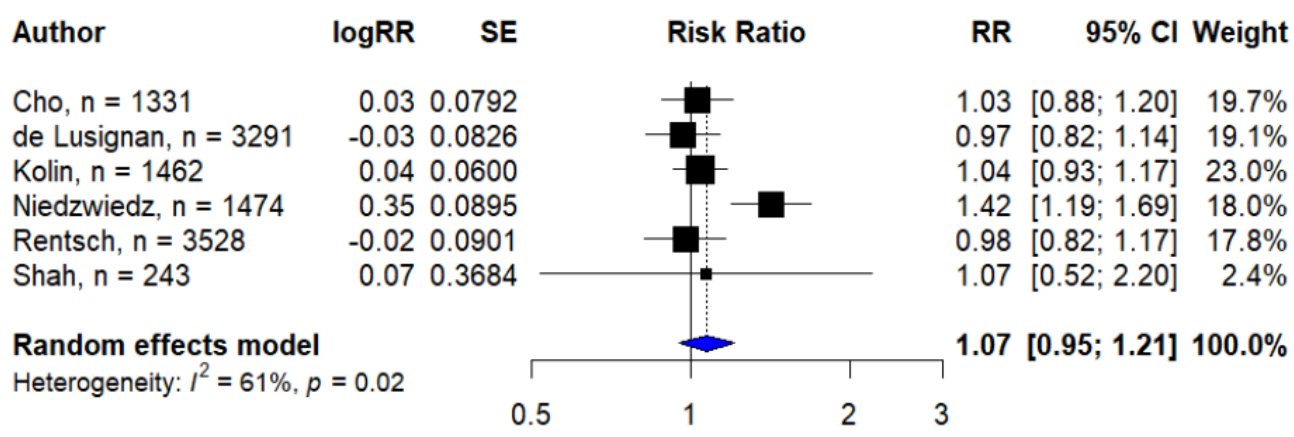

Figure 4. Forest plot for risk of testing positive for SARS-CoV-2 in former vs. never smokers.

Hospitalisation for COVID-19 by smoking status

Nine studies examined hospitalisation for COVID-19 disease stratified by smoking status (see Table 3). Meta-analyses were performed for five 'fair' quality studies. There was no significant difference between current and never smokers $(R R=1.12,95 \% \mathrm{Cl}=0.74-1.69$, $p=.48)$ or former and never smokers $(R R=1.21,95 \% \mathrm{Cl}=0.82-1.79, p=.24)$ in the risk of requiring admission to hospital following diagnosis of COVID-19 (see Figure 5 and 6, respectively). 


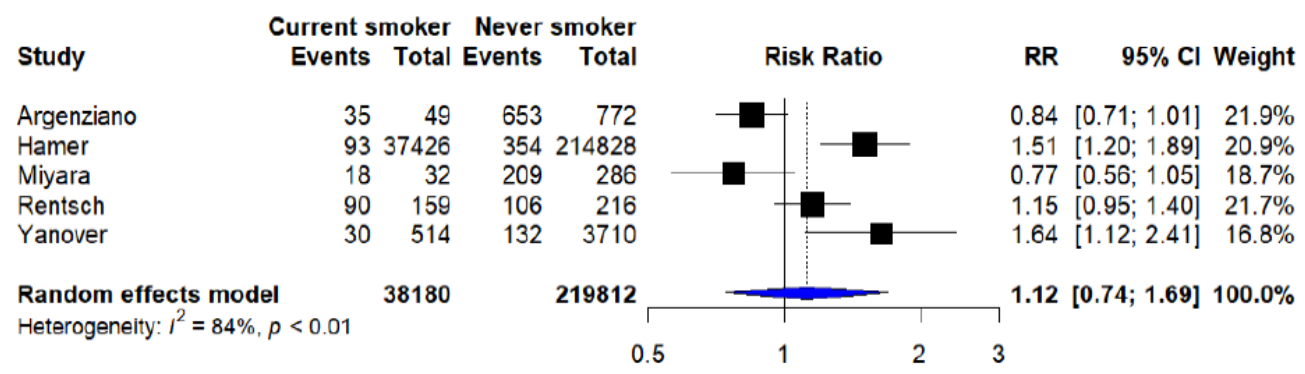

Figure 5. Forest plot for risk of hospitalisation in current vs. never smokers.

\begin{tabular}{|c|c|c|c|c|c|c|c|c|}
\hline \multirow[b]{2}{*}{ Study } & \multicolumn{2}{|c|}{ Former smoker } & \multicolumn{2}{|c|}{ Never smoker } & \multirow[b]{2}{*}{ Risk Ratio } & \multirow[b]{2}{*}{$\mathbf{R R}$} & \multirow[b]{2}{*}{$95 \% \mathrm{Cl}$} & \multirow[b]{2}{*}{ Weight } \\
\hline & Events & Total & Events & Total & & & & \\
\hline Argenziano & 161 & 179 & 653 & 772 & & 1.06 & {$[1.00 ; 1.13]$} & $23.1 \%$ \\
\hline Hamer & 313 & 134855 & 354 & 214828 & & 1.41 & {$[1.21 ; 1.64]$} & $21.9 \%$ \\
\hline Miyara & 111 & 152 & 209 & 286 & & 1.00 & {$[0.89 ; 1.13]$} & $22.4 \%$ \\
\hline Rentsch & 89 & 179 & 106 & 216 & & 1.01 & {$[0.83 ; 1.24]$} & $20.9 \%$ \\
\hline Yanover & 11 & 129 & 132 & 3710 & & 2.40 & {$[1.33 ; 4.32]$} & $11.6 \%$ \\
\hline \multirow{2}{*}{\multicolumn{2}{|c|}{$\begin{array}{l}\text { Random effects model } \\
\text { Heterogeneity: } I^{2}=81 \%, p<0.01\end{array}$}} & \multirow[t]{2}{*}{135494} & \multicolumn{2}{|r|}{219812} & & \multirow{2}{*}{\multicolumn{2}{|c|}{$1.21[0.82 ; 1.79]$}} & \multirow[t]{2}{*}{$100.0 \%$} \\
\hline & & & & 0. & 2 & & & \\
\hline
\end{tabular}

Figure 6. Forest plot for risk of hospitalisation in former vs. never smokers.

\section{Disease severity by smoking status}

Twenty-two studies reported disease severity in hospitalised patients stratified by smoking status (see Table 4). Severe (as opposed to non-severe) disease as broadly defined as requiring ITU admission, requiring oxygen as a hospital inpatient or in-hospital death (where this had not been disaggregated into disease severity vs. mortality). Metaanalyses were performed for three 'fair' quality studies. Current smokers were at increased risk of greater severity disease compared with never smokers ( $R R=1.37,95 \%$ $\mathrm{Cl}=1.07-1.75, \mathrm{p}=.01)$. No significant difference was observed between former and never smokers ( $R R=1.51,95 \% \mathrm{Cl}=0.82-2.80, p=.19)$ (see Figure 7 and 8 , respectively). 


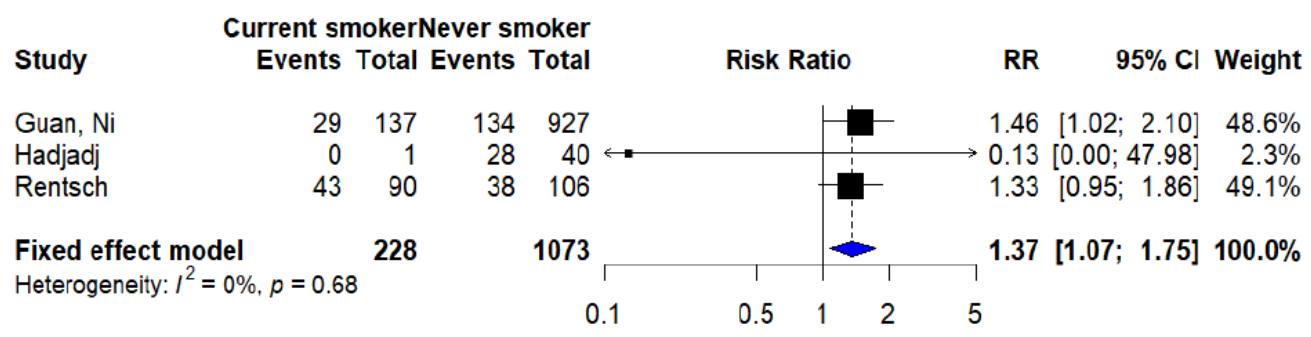

Figure 7. Forest plot for the risk of severe disease in current vs. never smokers.

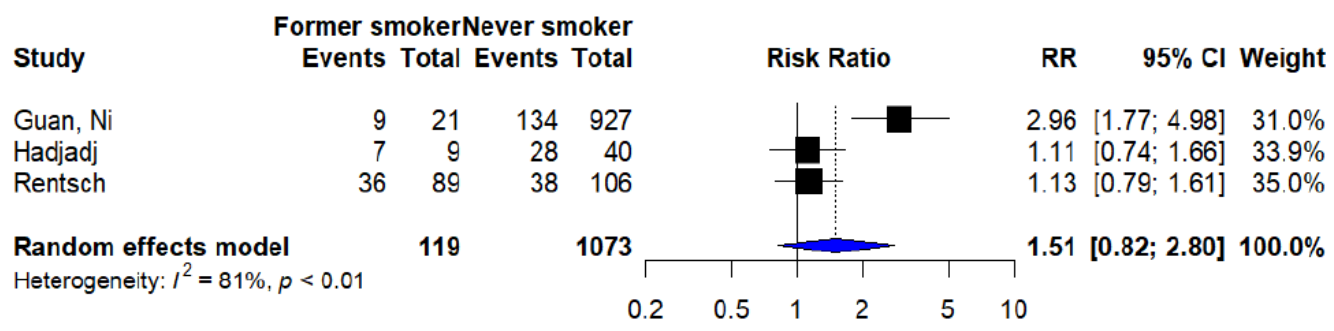

Figure 8. Forest plot for the risk of severe disease in former vs. never smokers.

Mortality by smoking status

Eleven studies reported mortality from COVID-19 by smoking status (see Table 6), with three 'fair' quality studies $66,80,82$. In the first study ${ }^{82}$, no significant difference in mortality was observed between current and never $(\mathrm{RR}=1.36,95 \% \mathrm{Cl}=0.85-2.17, \mathrm{p}=.24)$ or between former and never smokers ( $R R=0.91,95 \% \mathrm{Cl}=0.58-1.43, \mathrm{p}=.66)$. The second study ${ }^{66}$ reported hazard ratios adjusted for age and sex, suggesting an increased hazard of death in former $(\mathrm{HR}=1.80,95 \% \mathrm{Cl}=1.70-1.90)$ and current $(\mathrm{HR}=1.25,95 \% \mathrm{Cl}=1.12$ 1.40) compared with never smokers. In the adjusted primary analysis, the hazard in former smokers remained heightened $(\mathrm{HR}=1.25,95 \% \mathrm{Cl}=1.18-1.33)$ but reversed in current smokers ( $\mathrm{HR}=0.88,95 \% \mathrm{Cl}=0.79-0.99)$. The result was not robust in unplanned sensitivity analyses including further adjustment for ethnicity, early censoring and complete data for smoking and BMI. The third study ${ }^{80}$ reported odds ratios adjusted for age, sex, comorbidities and medication use, indicating increased odds of in-hospital death in current compared with never smokers $(\mathrm{OR}=1.79,95 \% \mathrm{Cl}=1.29-2.47)$.

\section{Quality appraisal}

Quality ratings for the included studies are presented in T able 7. Seventeen studies were rated as 'fair' quality due to having low levels of missing data and either i) distinguished 
between current, former and never smoking status or ii) adjusted analyses for potential confounders. The remaining 50 studies were rated as 'poor' quality.

Table 2. SARS-CoV-2 infection by smoking status.

\begin{tabular}{|c|c|c|c|c|c|c|c|c|c|c|c|c|c|}
\hline & $\begin{array}{l}\text { Total } \\
\text { population } \\
\text { tested }\end{array}$ & $\begin{array}{l}\text { SARS-CoV- } \\
2 \text { negative }\end{array}$ & & & & & & $\begin{array}{l}\text { SARS- } \\
\text { CoV-2 } \\
\text { positive }\end{array}$ & & & & & \\
\hline Author & $N$ & $\mathrm{~N}$ & $\begin{array}{l}\text { Current } \\
\text { smoker }\end{array}$ & $\begin{array}{l}\text { Former } \\
\text { smoker }\end{array}$ & $\begin{array}{l}\text { Current/former } \\
\text { smoker }\end{array}$ & $\begin{array}{l}\text { Never } \\
\text { smoker }\end{array}$ & $\begin{array}{l}\text { Not } \\
\text { stated }\end{array}$ & $\mathrm{N}$ & $\begin{array}{l}\text { Current } \\
\text { smoker }\end{array}$ & $\begin{array}{l}\text { Former } \\
\text { smoker }\end{array}$ & $\begin{array}{l}\text { Current/former } \\
\text { smoker }\end{array}$ & $\begin{array}{l}\text { Never } \\
\text { smoker }\end{array}$ & $\begin{array}{l}\text { Not } \\
\text { stated }\end{array}$ \\
\hline Rentsch & $3528^{*}$ & $\begin{array}{l}2974^{*} \\
(84.3 \%)\end{array}$ & $\begin{array}{l}1444 \\
(48.6 \%)\end{array}$ & $\begin{array}{l}704 \\
(23.6 \%)\end{array}$ & - & $\begin{array}{l}826 \\
(27.8 \%)\end{array}$ & - & $\begin{array}{l}554^{*} \\
(15.7 \%)\end{array}$ & $\begin{array}{l}159 \\
(28.7 \%)\end{array}$ & $\begin{array}{l}179 \\
(32.3 \%)\end{array}$ & - & $\begin{array}{l}216 \\
(39 \%)\end{array}$ & - \\
\hline Fontanet & 661 & $\begin{array}{l}490 \\
(74.1 \%)\end{array}$ & $\begin{array}{l}64 \\
(13.1 \%)\end{array}$ & $0(0 \%)$ & & $\begin{array}{l}426 \\
(86.4 \%)\end{array}$ & & $\begin{array}{l}171 \\
(25.9 \%)\end{array}$ & $5(2.9 \%)$ & $0(0 \%)$ & & $\begin{array}{l}166 \\
(97.1 \%)\end{array}$ & \\
\hline cho & 1331 & $\begin{array}{l}793 \\
(59.6 \%)\end{array}$ & $\begin{array}{l}142 \\
(17.9 \%)\end{array}$ & $\begin{array}{l}214 \\
(27 \%)\end{array}$ & - & $\begin{array}{l}437 \\
(55.1 \%)\end{array}$ & - & $\begin{array}{l}538 \\
(40.4 \%)\end{array}$ & $\begin{array}{l}111 \\
(20.6 \%)\end{array}$ & $\begin{array}{l}145 \\
(27 \%)\end{array}$ & & $\begin{array}{l}282 \\
(52.4 \%)\end{array}$ & - \\
\hline Shah & $243^{* *}$ & $\begin{array}{l}212 \\
(87.2 \%)\end{array}$ & $\begin{array}{l}52 \\
(24.5 \%)\end{array}$ & $\begin{array}{l}47 \\
(22.2 \%)\end{array}$ & - & $\begin{array}{l}113 \\
(53.3 \%)\end{array}$ & - & $\begin{array}{l}29 \\
(10.4 \%)\end{array}$ & $0(0 \%)$ & $9(31 \%)$ & - & $\begin{array}{l}20 \\
(69 \%)\end{array}$ & - \\
\hline $\begin{array}{l}\text { Bello- } \\
\text { Chavollo }\end{array}$ & 62,489 & $\begin{array}{l}46,960 \\
(75.2 \%)\end{array}$ & & & $4831(10.3 \%)$ & & $\begin{array}{l}42,125 \\
(89.7 \%)\end{array}$ & $\begin{array}{l}15,529 \\
(24.9 \%)\end{array}$ & & & $1374(8.8 \%)$ & & $\begin{array}{l}14,155 \\
(91.2 \%)\end{array}$ \\
\hline Kolin & $1474^{* * * *}$ & $\begin{array}{l}805 \\
(54.6 \%)\end{array}$ & $\begin{array}{l}141 \\
(17.5 \%)\end{array}$ & $\begin{array}{l}307 \\
(38.1 \%)\end{array}$ & - & $\begin{array}{l}354 \\
(44 \%)\end{array}$ & - & $\begin{array}{l}669 \\
(45.4 \%)\end{array}$ & $\begin{array}{l}72 \\
(10.8 \%)\end{array}$ & $\begin{array}{l}285 \\
(42.6 \%)\end{array}$ & - & $\begin{array}{l}303 \\
(45.3 \%)\end{array}$ & - \\
\hline
\end{tabular}

Note. Niedzwiedz et al. reported on SARS-CoV-2 infection by smoking status in multivariable analyses but did not present raw data; * Data on smoking status were missing

for 261 participants; ** Data on smoking status were missing for 75 participants; *** Data on smoking status were missing for 12 participants.

Table 3. Hospitalisation for COVID-19 by smoking status.

\begin{tabular}{|c|c|c|c|c|c|c|c|c|c|c|c|c|c|c|c|}
\hline \multicolumn{2}{|c|}{ COVID +ve sample } & \multicolumn{7}{|c|}{ community } & \multicolumn{7}{|c|}{ Hospitalised } \\
\hline Author & $\mathrm{N}$ & $\mathrm{N}$ & $\begin{array}{l}\text { Current } \\
\text { smoker }\end{array}$ & $\begin{array}{l}\text { Former } \\
\text { smoker }\end{array}$ & $\begin{array}{l}\text { Current/former } \\
\text { smoker }\end{array}$ & $\begin{array}{l}\text { Never } \\
\text { smoker }\end{array}$ & Never/unknown & $\begin{array}{l}\text { Not } \\
\text { stated }\end{array}$ & $\mathrm{N}$ & $\begin{array}{l}\text { Current } \\
\text { smoker }\end{array}$ & $\begin{array}{l}\text { Former } \\
\text { smoker }\end{array}$ & $\begin{array}{l}\text { Current/former } \\
\text { smoker }\end{array}$ & $\begin{array}{l}\text { Never } \\
\text { smoker }\end{array}$ & Never/unknown & $\begin{array}{l}\begin{array}{l}\text { Not } \\
\text { stated }\end{array} \\
\text { stal }\end{array}$ \\
\hline Rentsch & $554^{*}$ & $\begin{array}{l}269 \\
(48.6 \%)\end{array}$ & $\begin{array}{l}69 \\
(25.7 \%)\end{array}$ & $\begin{array}{l}90 \\
(33.5 \%)\end{array}$ & . & $\begin{array}{l}110 \\
(40.8 \%)\end{array}$ & - & - & $\begin{array}{l}285 \\
(51.4 \%)\end{array}$ & $\begin{array}{l}90 \\
(31.6 \%)\end{array}$ & $\begin{array}{l}89 \\
(31.2 \%)\end{array}$ & - & $\begin{array}{l}106 \\
(37.2 \%)\end{array}$ & - & . \\
\hline Petrilli & 4103 & $\begin{array}{l}2104 \\
(51.3 \%)\end{array}$ & $\begin{array}{l}108 \\
(5.1 \%)\end{array}$ & $\begin{array}{l}250 \\
(11.9 \%)\end{array}$ & . & - & $1746(83.0 \%)$ & - & $\begin{array}{l}1999 \\
(48.7 \%)\end{array}$ & $\begin{array}{l}104 \\
(5.2 \%)\end{array}$ & $\begin{array}{l}416 \\
(20.8 \%)\end{array}$ & - & - & $1479(74.0 \%)$ & . \\
\hline $\begin{array}{l}\text { Chow (US } \\
\text { CDC) }\end{array}$ & $6637^{\prime}$ & $\begin{array}{l}5143 \\
(77.5 \%)\end{array}$ & $\begin{array}{l}61 \\
(1.2 \%)\end{array}$ & $\begin{array}{l}80 \\
(1.6 \%)\end{array}$ & & & & $\begin{array}{l}5002 \\
(97.3 \%)\end{array}$ & $\begin{array}{l}1494 \\
(22.5 \%)\end{array}$ & $\begin{array}{l}27 \\
(1.8 \%)\end{array}$ & $\begin{array}{l}78 \\
(5.2 \%)\end{array}$ & & & & $\begin{array}{l}1389 \\
(93.0 \%)\end{array}$ \\
\hline Miyara & $482^{+*}$ & $\begin{array}{l}139 \\
(29.6 \%)\end{array}$ & $\begin{array}{l}14 \\
(10.1 \%)\end{array}$ & $\begin{array}{l}41 \\
(29.5 \%)\end{array}$ & & $\begin{array}{l}77 \\
(55.4 \%)\end{array}$ & - & $7(5 \%)$ & $\begin{array}{l}340 \\
(72.3 \%)\end{array}$ & $\begin{array}{l}18 \\
(5.3 \%)\end{array}$ & $\begin{array}{l}111 \\
(32.6 \%)\end{array}$ & & $\begin{array}{l}209 \\
(61.5 \%)\end{array}$ & & $\begin{array}{l}2 \\
(0.6 \%)\end{array}$ \\
\hline Argenziano & 1000 & $\begin{array}{l}151^{\wedge} \\
(15.1 \%)\end{array}$ & $\begin{array}{l}14 \\
(9.3 \%)\end{array}$ & $\begin{array}{l}18 \\
(11.9 \%)\end{array}$ & & $\begin{array}{l}119 \\
(78.8 \%)\end{array}$ & & & $\begin{array}{l}849 \\
(84.9 \%)\end{array}$ & $\begin{array}{l}35 \\
(4.1 \%)\end{array}$ & $\begin{array}{l}161 \\
(19.0 \%)\end{array}$ & & $\begin{array}{l}653 \\
(76.9 \%)\end{array}$ & & \\
\hline Lubetzky & 54 & $\begin{array}{l}15 \\
(27.8 \%)\end{array}$ & - & - & $4(26.7 \%)$ & - & - & $\begin{array}{l}11 \\
(73.3 \%)\end{array}$ & $\begin{array}{l}39 \\
(72.2 \%)\end{array}$ & - & - & $8(20.5 \%)$ & - & - & $\begin{array}{l}31 \\
(79.5 \%)\end{array}$ \\
\hline $\begin{array}{l}\text { Carrillo- } \\
\text { vega }\end{array}$ & 9946 & $\begin{array}{l}3922 \\
(39.4 \%)\end{array}$ & $\begin{array}{l}408 \\
(10.4 \%)\end{array}$ & - & & - & - & $\begin{array}{l}3514 \\
(89.6 \%)\end{array}$ & $\begin{array}{l}6024 \\
(60.6 \%)\end{array}$ & $\begin{array}{l}486 \\
(8.1 \%)\end{array}$ & & - & & - & $\begin{array}{l}5538 \\
(91.9 \%)\end{array}$ \\
\hline Yanover & 4353 & $\begin{array}{l}4180 \\
(96 \%)\end{array}$ & $\begin{array}{l}484 \\
(11.6 \%)\end{array}$ & $\begin{array}{l}118 \\
(2.8 \%)\end{array}$ & - & $\begin{array}{l}3578 \\
(85.6 \%)\end{array}$ & . & - & $\begin{array}{l}173 \\
(4 \%)\end{array}$ & $\begin{array}{l}30 \\
(17.3 \%)\end{array}$ & $\begin{array}{l}11 \\
(6.4 \%)\end{array}$ & - & $\begin{array}{l}132 \\
(76.3 \%)\end{array}$ & - & - \\
\hline Hamer & 387,109 & $\begin{array}{l}386,349 \\
(99,8 \% 6)\end{array}$ & $\begin{array}{l}37,333 \\
(9.7 \%)\end{array}$ & $\begin{array}{l}134,542 \\
(34.8 \% 6)\end{array}$ & - & $\begin{array}{l}214,474 \\
(55,6 \% 6)\end{array}$ & . & - & $\begin{array}{l}760 \\
(0.2 \%)\end{array}$ & $\begin{array}{l}93 \\
(12.2 \%)\end{array}$ & $\begin{array}{l}313 \\
(41.2 \%)\end{array}$ & . & $\begin{array}{l}354 \\
(46.6 \%)\end{array}$ & - & - \\
\hline
\end{tabular}


Table 4. Disease severity by smoking status.

\begin{tabular}{|c|c|c|c|c|c|c|c|c|c|c|c|c|c|c|c|}
\hline & $\begin{array}{c}\text { Sample } \\
\text { size }\end{array}$ & Non-sever & disease & & & & & & Severe di & iease & & & & & \\
\hline Author & $\mathrm{N}$ & $\mathrm{n}$ & $\begin{array}{l}\text { Current } \\
\text { smoker }\end{array}$ & $\begin{array}{l}\begin{array}{l}\text { Former } \\
\text { smoker }\end{array} \\
\text { s }\end{array}$ & $\begin{array}{l}\text { Current/former } \\
\text { smoker }\end{array}$ & $\begin{array}{l}\text { Never } \\
\text { smoker }\end{array}$ & $\begin{array}{l}\text { Never } \\
\text { smoker/unknown }\end{array}$ & $\begin{array}{l}\text { Not } \\
\text { stated }\end{array}$ & $\mathrm{n}$ & $\begin{array}{l}\text { Current } \\
\text { smoker }\end{array}$ & $\begin{array}{l}\text { Fomer } \\
\text { smoker }\end{array}$ & $\begin{array}{l}\text { Current/former } \\
\text { smoker }\end{array}$ & $\begin{array}{l}\text { Never } \\
\text { smoker }\end{array}$ & $\begin{array}{l}\text { Never } \\
\text { smoker/unknown }\end{array}$ & $\begin{array}{l}\text { Not } \\
\text { stated }\end{array}$ \\
\hline Guan, Ni & $1085^{\circ}$ & $\begin{array}{l}913 \\
\text { (84.1\%) }\end{array}$ & $\begin{array}{l}108 \\
(11.8 \%)\end{array}$ & $\begin{array}{l}12 \\
(1.3 \%)\end{array}$ & - & $\begin{array}{l}793 \\
(86.9 \%)\end{array}$ & - & - & $\begin{array}{l}172 \\
(15.9 \%)\end{array}$ & $\begin{array}{l}29 \\
(16.9 \%)\end{array}$ & $\stackrel{9}{(5.2 \%)}$ & - & $\begin{array}{l}134 \\
(77.9 \%)\end{array}$ & $\cdot$ & . \\
\hline $\begin{array}{l}\text { Zhang, } \\
\text { Dong }\end{array}$ & $9^{\circ}$ & $\begin{array}{l}3 \\
\text { (33.3\%) }\end{array}$ & $0(0.0 \%)$ & $\begin{array}{l}3 \\
(100.0 \%)\end{array}$ & - & - & - & - & $\begin{array}{l}6 \\
(66.7 \%)\end{array}$ & $\begin{array}{l}2 \\
\text { (33.3\%) }\end{array}$ & $\begin{array}{l}4 \\
(66.7 \%)\end{array}$ & - & - & - & - \\
\hline Wan & $9^{c}$ & $\begin{array}{l}8 \\
(88.9 \%)\end{array}$ & $\begin{array}{l}8 \\
(100.0 \%)\end{array}$ & - & - & - & - & - & ${ }_{(11.1 \%)}^{1}$ & $\begin{array}{l}1 \\
(100.0 \%)\end{array}$ & - & - & - & - & - \\
\hline $\begin{array}{l}\text { Huang, } \\
\text { Wang }\end{array}$ & $3^{a}$ & $\begin{array}{l}3 \\
(100.0 \%)\end{array}$ & $\begin{array}{l}3 \\
(100.0 \%)\end{array}$ & & - & - & - & - & $\begin{array}{l}0 \\
(0.0 \%)\end{array}$ & $0(0.0 \%)$ & - & - & - & - & - \\
\hline Rentsch & 285 & $\begin{array}{l}168 \\
(58.9 \%)^{*}\end{array}$ & $\begin{array}{l}47 \\
(28.0 \%)\end{array}$ & $\begin{array}{l}53 \\
(31.5 \%)\end{array}$ & - & $\begin{array}{l}68 \\
(40.4 \%)\end{array}$ & - & - & $\begin{array}{l}117 \\
(21.1 \%)\end{array}$ & $\begin{array}{l}43 \\
(36.8 \%)\end{array}$ & $\begin{array}{l}36 \\
(30.8 \%)\end{array}$ & - & $\begin{array}{l}38 \\
(32.5 \%)\end{array}$ & - & - \\
\hline Hu & 323 & $\begin{array}{l}151 \\
(46.7 \%)\end{array}$ & - & - & $12(7.9 \%)$ & - & - & $\begin{array}{l}139 \\
(92.1 \%)\end{array}$ & $\begin{array}{l}172 \\
(53.3 \%)\end{array}$ & - & - & $26(15.1 \%)$ & - & - & $\begin{array}{l}146 \\
(84.9 \%)\end{array}$ \\
\hline Wang, Pan & 125 & $\begin{array}{l}100 \\
(80.0 \%)\end{array}$ & - & - & 9 (9.0\%) & - & - & $\begin{array}{l}91 \\
\text { (91.0\%) }\end{array}$ & $\begin{array}{l}25 \\
(20.0 \%)\end{array}$ & - & - & $7(28.0 \%)$ & - & - & $\begin{array}{l}18 \\
(72.0 \%)\end{array}$ \\
\hline Petrilli & 4103 & $\begin{array}{l}932 \\
(22.7 \%)^{*}\end{array}$ & $\begin{array}{l}62 \\
(6.7 \%)\end{array}$ & $\begin{array}{l}175 \\
(18.8 \%)\end{array}$ & - & - & 695 (74.6\%) & - & $\begin{array}{l}650 \\
(15.8 \%)\end{array}$ & $\begin{array}{l}28 \\
(4.3 \%)\end{array}$ & $\begin{array}{l}\text { 145 } \\
(22.3 \%)\end{array}$ & - & - & $477(73.4 \%)$ & - \\
\hline Kim & $27^{\circ}$ & $\begin{array}{l}21 \\
(81.5 \%)\end{array}$ & $\begin{array}{l}3 \\
(60.0 \%)\end{array}$ & - & - & - & $18(82.6 \%)$ & - & $\begin{array}{l}6 \\
(22.2 \%)\end{array}$ & $\begin{array}{l}2 \\
(40.0 \%)\end{array}$ & - & - & - & $4(17.4 \%)$ & - \\
\hline Shi, Yu & $474^{\circ}$ & $\begin{array}{l}425 \\
(89.7 \%)\end{array}$ & - & - & $34(7.8 \%)$ & - & 391 (89.3\%) & - & $\begin{array}{l}49 \\
(10.3 \%)\end{array}$ & - & & $6(12.2 \%)$ & - & $43(87.8 \%)$ & - \\
\hline Liao, Feng & 1485 & $\begin{array}{l}92 \\
(62.2 \%)\end{array}$ & - & - & $5(5.4 \%)$ & - & - & $\begin{array}{l}87 \\
(94.6 \%)\end{array}$ & $\begin{array}{l}56 \\
(37.8 \%)\end{array}$ & $3(5.4 \%)$ & - & - & - & - & $\begin{array}{l}53 \\
(94.6 \%)\end{array}$ \\
\hline Shi, Ren & 134 & $\begin{array}{l}88 \\
(65.7 \%)\end{array}$ & - & $8(9.1 \%)$ & - & - & - & $\begin{array}{l}80 \\
(90.9 \%)\end{array}$ & $\begin{array}{l}46 \\
(34.3 \%)\end{array}$ & - & - & $6(13.0 \%)$ & - & - & $\begin{array}{l}40 \\
(87.0 \%)\end{array}$ \\
\hline Hadjadj & 50 & $\begin{array}{l}15 \\
(30.0 \%)\end{array}$ & $1(6.7 \%)$ & $\begin{array}{l}2 \\
(13.3 \%)\end{array}$ & - & $\begin{array}{l}12 \\
(80.0 \%)\end{array}$ & - & - & $\begin{array}{l}35 \\
(70.0 \%)\end{array}$ & $0(0.0 \%)$ & $\begin{array}{l}7 \\
(20.0 \%)\end{array}$ & - & $\begin{array}{l}28 \\
(80 \%)\end{array}$ & - & - \\
\hline $\begin{array}{l}\text { Zheng, } \\
\text { Xiong }\end{array}$ & 73 & $\begin{array}{l}43 \\
(58.9 \%)\end{array}$ & - & - & $6(14 \%)$ & $\begin{array}{l}37 \\
(86 \%)\end{array}$ & & - & $\begin{array}{l}30 \\
(41.1 \%)\end{array}$ & - & & $2(6.7 \%)$ & $\begin{array}{l}28 \\
(93.3 \%)\end{array}$ & - & - \\
\hline de la Rica & 48 & $\begin{array}{l}26 \\
(54.2 \%)\end{array}$ & - & - & $6(23.1 \%)$ & - & - & $\begin{array}{l}20 \\
(76.9 \%)\end{array}$ & $\begin{array}{l}20 \\
(41.7 \%)\end{array}$ & - & - & $4(20 \%)$ & - & - & $\begin{array}{l}16 \\
(80 \%)\end{array}$ \\
\hline
\end{tabular}

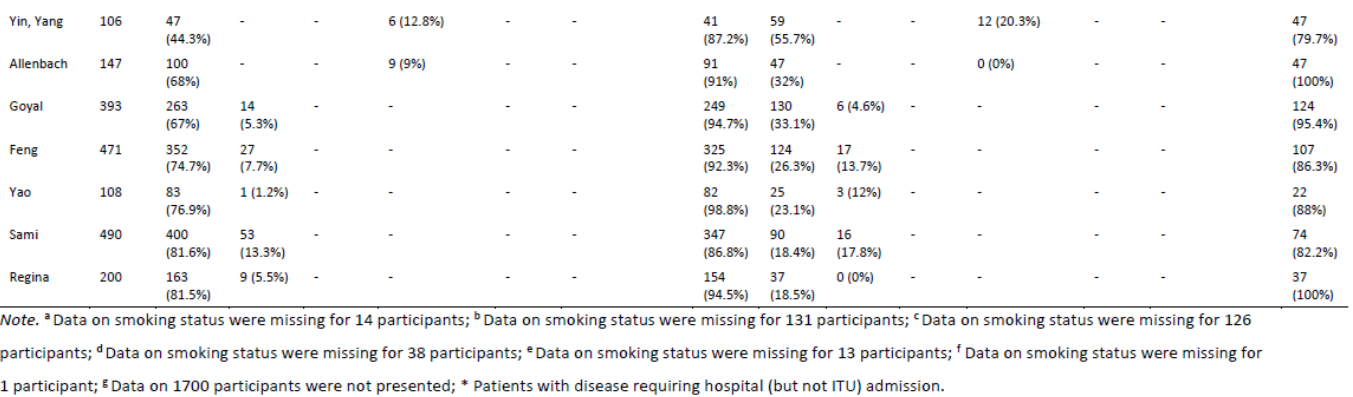

Table 5 . Mortality by smoking status.

\begin{tabular}{|c|c|c|c|c|c|c|c|c|c|c|c|c|c|c|c|}
\hline \multicolumn{2}{|c|}{ Sample size } & \multicolumn{7}{|l|}{ Death } & \multicolumn{7}{|l|}{ Recovery } \\
\hline Author & $\mathrm{N}$ & $\mathrm{n}$ & $\begin{array}{l}\text { Current } \\
\text { smoker }\end{array}$ & $\begin{array}{l}\text { Former } \\
\text { smoker }\end{array}$ & $\begin{array}{l}\text { Current/former } \\
\text { smoker }\end{array}$ & $\begin{array}{l}\text { Never } \\
\text { smoker }\end{array}$ & $\begin{array}{l}\text { Never } \\
\text { smoker/unknown }\end{array}$ & $\begin{array}{l}\text { Not } \\
\text { stated }\end{array}$ & $\mathrm{n}$ & $\begin{array}{l}\text { Current } \\
\text { smoker }\end{array}$ & $\begin{array}{l}\begin{array}{l}\text { Former } \\
\text { smoker }\end{array} \\
\end{array}$ & $\begin{array}{l}\text { Current/former } \\
\text { smoker }\end{array}$ & $\begin{array}{l}\text { Never } \\
\text { smoker }\end{array}$ & $\begin{array}{l}\text { Never } \\
\text { smoker/unknown }\end{array}$ & $\begin{array}{l}\text { Not } \\
\text { stated }\end{array}$ \\
\hline chen & $274^{*}$ & $\begin{array}{l}113 \\
(41.2 \%)\end{array}$ & $\begin{array}{l}7 \\
(6.2 \%)\end{array}$ & $\stackrel{2}{(1.8 \%)}$ & . & - & - & $\begin{array}{l}104 \\
(92.0 \%)^{\wedge}\end{array}$ & $\begin{array}{l}161 \\
(58.8)\end{array}$ & $\begin{array}{l}5 \\
(3.1 \%)\end{array}$ & - & - & - & - & $\begin{array}{l}156 \\
(96.9 \%)\end{array}$ \\
\hline zhou & 191 & $\begin{array}{l}54 \\
(28.3 \%)\end{array}$ & $\begin{array}{l}5 \\
(9.3 \%)\end{array}$ & - & - & - & - & $\begin{array}{l}49 \\
(90.706)\end{array}$ & $\begin{array}{l}137 \\
(71.796)\end{array}$ & $\begin{array}{l}6 \\
(4.4 \%)\end{array}$ & - & - & - & - & $\begin{array}{l}131 \\
\text { (95.6\%) }\end{array}$ \\
\hline Yang, Yu & 52 & $\begin{array}{l}32 \\
(61.5 \%)\end{array}$ & $\begin{array}{l}0 \\
(0.0 \%)\end{array}$ & - & - & - & $32(100.0 \%)$ & - & $\begin{array}{l}20 \\
(38.5 \%)\end{array}$ & $\begin{array}{l}2 \\
(10.0 \%)\end{array}$ & - & - & - & $18(90.0 \% 6)$ & - \\
\hline Mehra & 8910 & $\begin{array}{l}515 \\
(5.8 \%)\end{array}$ & $\begin{array}{l}46 \\
(8.9 \%)\end{array}$ & $\begin{array}{l}83 \\
(16.1 \%)\end{array}$ & - & - & - & $386(4.3$ & $\begin{array}{l}8395 \\
(94.2 \%)\end{array}$ & $\begin{array}{l}445 \\
(5.3 \%)\end{array}$ & $\begin{array}{l}1410 \\
(16.8 \%)\end{array}$ & - & - & - & $\begin{array}{l}6540 \\
(77.9 \%)\end{array}$ \\
\hline Gaibazzi & 441 & $\begin{array}{l}156 \\
(35.4 \%)\end{array}$ & $\begin{array}{l}10 \\
(6.4 \%)\end{array}$ & $14(9 \%)$ & - & $\begin{array}{l}132 \\
(84.6 \%)\end{array}$ & - & - & $\begin{array}{l}285 \\
(64.6 \%)\end{array}$ & $\begin{array}{l}11 \\
(3.9 \%)\end{array}$ & $\begin{array}{l}30 \\
(10.5 \%)\end{array}$ & - & $\begin{array}{l}244 \\
(85.6 \%)\end{array}$ & - & - \\
\hline Borobia & 2226 & $\begin{array}{l}460 \\
(20.7 \%)\end{array}$ & $\begin{array}{l}77 \\
(9.6 \%)\end{array}$ & - & - & - & - & $\begin{array}{l}416 \\
(90.4 \%)\end{array}$ & $\begin{array}{l}1766 \\
(79.3 \%)\end{array}$ & $\begin{array}{l}113 \\
(6.4 \%)\end{array}$ & - & - & - & - & $\begin{array}{l}1653 \\
(93.6 \%)\end{array}$ \\
\hline Giacomelli & 233 & $\begin{array}{l}48 \\
(20.6 \%)\end{array}$ & - & . & $17(35.4 \%)$ & $\begin{array}{l}31 \\
(64.6 \%)\end{array}$ & . & - & $\begin{array}{l}185 \\
(79.4 \%)\end{array}$ & - & - & $53(28.6 \%)$ & $\begin{array}{l}132 \\
(71.4 \%)\end{array}$ & & \\
\hline Yao & 108 & $\begin{array}{l}12 \\
(11.1 \%)\end{array}$ & $3(25 \%)$ & - & - & - & - & $9(75 \%)$ & $\begin{array}{l}96 \\
(88.9 \%)\end{array}$ & $1(1 \%)$ & - & - & - & - & $\begin{array}{l}95 \\
(99 \%)\end{array}$ \\
\hline $\begin{array}{l}\text { Carrillo- } \\
\text { vega }\end{array}$ & 9946 & $\begin{array}{l}963 \\
(9.7 \% 6)\end{array}$ & $\begin{array}{l}99 \\
(10.3 \%)\end{array}$ & - & - & - & - & $\begin{array}{l}864 \\
(89.7 \%)\end{array}$ & $\begin{array}{l}8983 \\
(90.3 \%)\end{array}$ & $\begin{array}{l}795 \\
(8.9 \%)\end{array}$ & - & - & - & - & $\begin{array}{l}8188 \\
(91.1 \%)\end{array}$ \\
\hline
\end{tabular}


Table 6. Quality ratings of included studies.

\begin{tabular}{|c|c|c|c|c|c|c|c|c|c|c|c|c|c|c|c|}
\hline Author & 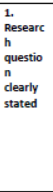 & 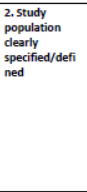 & $\begin{array}{l}\text { 3. Participation } \\
\text { rate of eligible } \\
\text { persons at } \\
\text { least } 50 \%\end{array}$ & 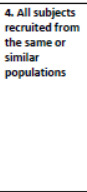 & $\begin{array}{l}\text { s.5sample } \\
\text { size } \\
\text { justificati } \\
\text { on } \\
\text { provided }\end{array}$ & $\begin{array}{l}\text { 6. Exposure of } \\
\text { interest } \\
\text { measured } \\
\text { periortod } \\
\text { outcome(s) }\end{array}$ & $\begin{array}{l}\text { 7.Timefiame } \\
\text { sutficient to } \\
\text { sean an } \\
\text { associaiation } \\
\text { between } \\
\text { exposure and } \\
\text { outcome if it } \\
\text { existed }\end{array}$ & $\begin{array}{l}8 . \\
\text { Examin } \\
\text { Exd } \\
\text { differen } \\
\text { televels } \\
\text { of the } \\
\text { exposur } \\
\text { easur } \\
\text { related } \\
\text { tothe } \\
\text { outcom } \\
\text { e }\end{array}$ & $\begin{array}{l}9 . \\
\text { Exposu } \\
\text { re } \\
\text { measur } \\
\text { e } \\
\text { clearly } \\
\text { defined } \\
\text {,valid } \\
\text { and } \\
\text { reliable }\end{array}$ & 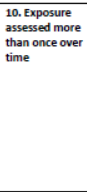 & $\begin{array}{l}\text { I1. } \\
\text { outcome } \\
\text { measurere } \\
\text { s) dearly } \\
\text { defined, } \\
\text { valid and } \\
\text { reliable }\end{array}$ & $\begin{array}{l}12 . \\
\text { outcom } \\
\text { essesso } \\
\text { ass } \\
\text { rs } \\
\text { blinded } \\
\text { to } \\
\text { exposur } \\
\text { e status }\end{array}$ & $\begin{array}{l}\text { 13. Loss to } \\
\text { follow-up } \\
\text { atter } \\
\text { baseline } \\
\text { 20\%5 or less }\end{array}$ & 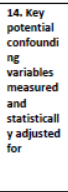 & $\begin{array}{l}\text { Oover } \\
\text { Irtating }\end{array}$ \\
\hline Guan, Ni & Yes & No & No & $\begin{array}{l}\text { Cannot } \\
\text { determin }\end{array}$ & No & Yes & $\begin{array}{l}\text { cannot } \\
\text { determine }\end{array}$ & Yes & No & $\begin{array}{l}\text { Cannot } \\
\text { determin }\end{array}$ & ves & No & $\begin{array}{l}\text { Not } \\
\text { appopicable }\end{array}$ & No & Fair \\
\hline Guan, Liang & res & No & No & $\begin{array}{l}\text { cannot } \\
\text { determines }\end{array}$ & No & yes & $\begin{array}{l}\text { cannot } \\
\text { deteretine }\end{array}$ & № & No & $\begin{array}{l}\text { canoot } \\
\text { determing }\end{array}$ & res & No & $\begin{array}{l}\text { Not } \\
\text { anopizable }\end{array}$ & res & Fair \\
\hline Lian & ves & No & $\begin{array}{l}\text { Cannot } \\
\text { determinn }\end{array}$ & $\begin{array}{l}\text { Cannot } \\
\text { determine }\end{array}$ & No & yes & $\begin{array}{l}\text { cannot } \\
\text { determine }\end{array}$ & No & No & $\begin{array}{l}\text { Cannot } \\
\text { determinn }\end{array}$ & ves & No & $\begin{array}{l}\text { Not } \\
\text { applicable }\end{array}$ & No & Poor \\
\hline Jin & res & res & $\begin{array}{l}\text { cannot } \\
\text { determing }\end{array}$ & $\begin{array}{l}\text { Cannot } \\
\text { dateremint }\end{array}$ & No & yes & $\begin{array}{l}\text { cannot } \\
\text { cetermine }\end{array}$ & No & No & $\begin{array}{l}\text { Cannot } \\
\text { doterming }\end{array}$ & res & No & $\begin{array}{l}\text { Not } \\
\text { Not }\end{array}$ & No & poor \\
\hline chen & res & res & $\begin{array}{l}\text { Cechnot } \\
\text { determine }\end{array}$ & Yes & No & ves & $\begin{array}{l}\text { cennot } \\
\text { determine }\end{array}$ & yes & no & $\begin{array}{l}\text { Cannot } \\
\text { determin }\end{array}$ & ves & No & $\begin{array}{l}\text { Not } \\
\text { applicable }\end{array}$ & No & Poor \\
\hline zhou, ru & res & res & ves & res & No & res & $\begin{array}{l}\text { cannot } \\
\text { caterget }\end{array}$ & No & No & $\begin{array}{l}\text { Cannot } \\
\text { Canter }\end{array}$ & Yes & No & $\begin{array}{l}\text { Not } \\
\text { Not }\end{array}$ & No & Poor \\
\hline Mo & res & res & Cannot & res & No & Yes & 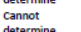 & No & No & 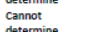 & ves & No & $\begin{array}{l}\text { Not } \\
\text { Noticue }\end{array}$ & No & Poor \\
\hline zhang, Dong & res & res & $\begin{array}{l}\text { Yes } \\
\text { Yolen }\end{array}$ & res & мо & res & $\begin{array}{l}\text { ceerernine } \\
\text { catert } \\
\text { determine }\end{array}$ & res & no & 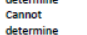 & Yes & No & $\begin{array}{l}\text { Nppot } \\
\text { Noplical }\end{array}$ & No & Poor \\
\hline Wan & res & No & $\begin{array}{c}\text { cannot } \\
\text { determin }\end{array}$ & $\begin{array}{l}\text { Cannot } \\
\text { determing }\end{array}$ & No & res & $\begin{array}{l}\text { Cannot } \\
\text { deterermine }\end{array}$ & No & No & $\begin{array}{l}\text { Cannot } \\
\text { determin }\end{array}$ & ves & No & Not & No & Poor \\
\hline Lü, тао & Yes & res & Yes & Yes & No & Cannot & cannot & No & No & Cannot & Yes & No & Not & No & Poor \\
\hline Huang, Wang & ves & res & yes & res & No & Yes & $\begin{array}{l}\text { cannot } \\
\text { deteretine }\end{array}$ & No & No & $\begin{array}{l}\text { Cannot } \\
\text { determin }\end{array}$ & ves & No & $\begin{array}{l}\text { Not } \\
\text { appopicable }\end{array}$ & No & Poor \\
\hline zhang, cai & res & No & $\begin{array}{l}\text { cannot } \\
\text { detretrin }\end{array}$ & res & No & $\begin{array}{l}\text { Cannot } \\
\text { daterminin }\end{array}$ & $\begin{array}{l}\text { Decermine } \\
\text { cantot } \\
\text { determine }\end{array}$ & No & no & $\begin{array}{l}\text { cetermin } \\
\text { cannot } \\
\text { deteremingin }\end{array}$ & res & No & $\begin{array}{l}\text { Npot } \\
\text { Noticle }\end{array}$ & No & poor \\
\hline Guo & res & res & $\begin{array}{l}\text { Oeter } \\
\text { ves }\end{array}$ & res & No & $\begin{array}{l}\text { Des } \\
\text { Yest }\end{array}$ & 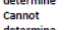 & No & no & 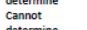 & ves & No & $\begin{array}{l}\text { pappiciole } \\
\text { Not }\end{array}$ & No & Poor \\
\hline Liu, Ming & res & res & Yes & res & No & res & $\begin{array}{l}\text { determinine } \\
\text { cannot } \\
\text { determinte }\end{array}$ & No & no & $\begin{array}{l}\text { determint } \\
\text { cannot } \\
\text { denet }\end{array}$ & Yes & No & $\begin{array}{l}\text { applicable } \\
\text { Not }\end{array}$ & No & Poor \\
\hline Huang, rang & res & res & cannot & Cannot & No & res & $\begin{array}{l}\text { cannot } \\
\text { cant } \\
\text { cons }\end{array}$ & No & мо & $\begin{array}{l}\text { detetminin } \\
\text { cannot }\end{array}$ & ves & No & $\begin{array}{l}\text { appicable } \\
\text { Not }\end{array}$ & o & Poor \\
\hline $\mathrm{xu}$ & res & res & 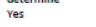 & ves & No & res & $\begin{array}{l}\text { Deermine } \\
\text { Cannot }\end{array}$ & No & No & $\begin{array}{l}\text { Determint } \\
\text { Cannot }\end{array}$ & ves & No & $\begin{array}{l}\text { Pappicidele } \\
\text { Not }\end{array}$ & No & Poor \\
\hline ü & res & No & cannot & Cannot & No & Yes & $\begin{array}{l}\text { determine } \\
\text { cannot } \\
\text { determitate }\end{array}$ & No & No & $\begin{array}{l}\text { detetmint } \\
\text { cannot } \\
\text { determin }\end{array}$ & ves & No & $\begin{array}{l}\text { applicable } \\
\text { Not }\end{array}$ & No & Poor \\
\hline Rentsch & Yes & res & $\begin{array}{l}\text { 然eter } \\
\text { Yes }\end{array}$ & $\begin{array}{l}\text { deter } \\
\text { Yes }\end{array}$ & мо & Yes & $\begin{array}{l}\text { deternmine } \\
\text { cannot }\end{array}$ & res & no & Cannot & ves & No & $\begin{array}{l}\text { applicable } \\
\text { Not }\end{array}$ & Yes & Fair \\
\hline Hu & res & No & Cannot & cannot & No & Yes & 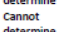 & No & No & $\begin{array}{l}\text { determine } \\
\text { cannot }\end{array}$ & yes & No & $\begin{array}{l}\text { appoicable } \\
\text { Not }\end{array}$ & ves & Fair \\
\hline
\end{tabular}

\begin{tabular}{|c|c|c|c|c|c|c|c|c|c|c|c|c|c|c|c|}
\hline Wang pan & res & No & $\begin{array}{l}\text { Cannot } \\
\text { datermine }\end{array}$ & $\begin{array}{l}\text { Cannot } \\
\text { datempine }\end{array}$ & No & Yes & $\begin{array}{l}\text { cannot } \\
\text { determine }\end{array}$ & No & № & $\begin{array}{l}\text { Cannot } \\
\text { datermine }\end{array}$ & Yes & No & Not & No & Poor \\
\hline Petrilli & Yes & ves & res & Yes & No & Yes & $\begin{array}{l}\text { cannot } \\
\text { caterg }\end{array}$ & yes & No & $\begin{array}{l}\text { cannot } \\
\text { cantertate }\end{array}$ & Yes & No & Not & Yes & Fair \\
\hline chow (US CDC) & res & No & No & No & No & res & $\begin{array}{l}\text { cannot } \\
\text { cante }\end{array}$ & No & No & cannot & Yes & No & $\begin{array}{l}\text { Not } \\
\text { Not }\end{array}$ & № & Poor \\
\hline Mivara & res & ves & res & res & No & res & $\begin{array}{l}\text { cernotine } \\
\text { cannt }\end{array}$ & yes & No & $\begin{array}{l}\text { Decermine } \\
\text { cannot }\end{array}$ & ves & No & $\begin{array}{l}\text { Sot } \\
\text { Not }\end{array}$ & No & Fair \\
\hline Dong, Cao & No & No & cannot & Cannot & No & yes & $\begin{array}{l}\text { canot } \\
\text { Cannet }\end{array}$ & No & No & $\begin{array}{l}\text { cannot } \\
\text { canter }\end{array}$ & Yes & No & $\begin{array}{l}\text { Soptoule } \\
\text { Not }\end{array}$ & No & Poor \\
\hline kim & res & ves & $\begin{array}{l}\text { ves } \\
\text { vertents }\end{array}$ & 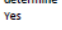 & No & Yes & $\begin{array}{l}\text { Cannot } \\
\text { Cons }\end{array}$ & No & No & 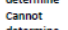 & ves & No & $\begin{array}{l}\text { Not } \\
\text { Not }\end{array}$ & No & Poor \\
\hline Shi, ru & res & ves & res & res & No & yes & $\begin{array}{l}\text { Decermine } \\
\text { Cannot }\end{array}$ & № & No & $\begin{array}{l}\text { Decermine } \\
\text { Cannot }\end{array}$ & yes & No & $\begin{array}{l}\text { Not } \\
\text { Not }\end{array}$ & № & Poor \\
\hline rang, yu & res & ves & res & res & No & res & 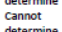 & No & No & 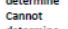 & yes & № & $\begin{array}{l}\text { Sapticable } \\
\text { Not }\end{array}$ & No & Poor \\
\hline Argenziano & res & ves & res & Yes & No & Yes & $\begin{array}{l}\text { Decermine } \\
\text { Cannot }\end{array}$ & Yes & No & $\begin{array}{l}\text { determine } \\
\text { cannot }\end{array}$ & Yes & No & $\begin{array}{l}\text { applicable } \\
\text { Not }\end{array}$ & No & Fair \\
\hline solis & res & No & Cannot & $\begin{array}{l}\text { cannot } \\
\text { deternine }\end{array}$ & No & res & $\begin{array}{l}\text { Determine } \\
\text { cannot }\end{array}$ & No & No & 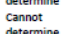 & res & No & $\begin{array}{l}\text { Jppirtale } \\
\text { Not }\end{array}$ & res & Poor \\
\hline Richardson & Yes & res & $\begin{array}{l}\text { Oetermine } \\
\text { Yes }\end{array}$ & $\begin{array}{l}\text { Determine } \\
\text { Yes }\end{array}$ & No & Yes & $\begin{array}{l}\text { determine } \\
\text { cannot }\end{array}$ & No & No & $\begin{array}{l}\text { oeterminine } \\
\text { cannot }\end{array}$ & Yes & No & $\begin{array}{l}\text { appictale } \\
\text { Not }\end{array}$ & No & Poor \\
\hline Fontanet & ves & ves & yes & res & No & No & 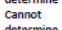 & № & No & No & yes & No & Not & No & Poor \\
\hline zheng, Gao & res & res & res & res & No & cannot & $\begin{array}{l}\text { cannot } \\
\text { catertint }\end{array}$ & No & No & cannot & ves & No & Not & No & Poor \\
\hline Liao, Feng & res & res & res & res & мо & $\begin{array}{l}\text { Determine } \\
\text { Cannot }\end{array}$ & $\begin{array}{l}\text { deermine } \\
\text { Cannot }\end{array}$ & № & No & $\begin{array}{l}\text { oeterminne } \\
\text { Cannot }\end{array}$ & res & No & $\begin{array}{l}\text { Sapticale } \\
\text { Not }\end{array}$ & No & Poor \\
\hline Rodriguez & ves & res & Yes & res & No & $\begin{array}{l}\text { Dester } \\
\text { Yyes }\end{array}$ & $\begin{array}{l}\text { determine } \\
\text { cannot }\end{array}$ & No & No & $\begin{array}{l}\text { determine } \\
\text { cannot } \\
\text { chent }\end{array}$ & Yes & No & $\begin{array}{l}\text { applicable } \\
\text { ves }\end{array}$ & No & Poor \\
\hline Magagnoli & res & res & res & res & No & yes & $\begin{array}{l}\text { Determine } \\
\text { canot } \\
\text { determinge }\end{array}$ & № & No & 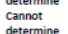 & yes & No & Not & No & Poor \\
\hline Shi, Ren & res & No & Cannot & cannot & No & res & cannot & No & No & Cannot & ves & No & $\begin{array}{l}\text { Sotictie } \\
\text { Not }\end{array}$ & No & Poor \\
\hline Hajjadj & res & мо & $\begin{array}{l}\text { determine } \\
\text { Cannot }\end{array}$ & $\begin{array}{l}\text { determinit } \\
\text { Cannot }\end{array}$ & мо & Cannot & $\begin{array}{l}\text { detetrmine } \\
\text { cannot }\end{array}$ & Yes & No & $\begin{array}{l}\text { determinine } \\
\text { cannot }\end{array}$ & Yes & No & $\begin{array}{l}\text { applicable } \\
\text { Not }\end{array}$ & No & Poor \\
\hline Niedzwiedz & res & ves & $\begin{array}{l}\text { determine } \\
\text { res }\end{array}$ & $\begin{array}{l}\text { determine } \\
\text { ves }\end{array}$ & No & $\begin{array}{l}\text { determine } \\
\text { yes }\end{array}$ & $\begin{array}{l}\text { determine } \\
\text { Cannot }\end{array}$ & yes & No & $\begin{array}{l}\text { determine } \\
\text { cannot }\end{array}$ & yes & No & $\begin{array}{l}\text { applicable } \\
\text { Not }\end{array}$ & Yes & Fair \\
\hline Gold (US CDC) & res & Yes & ves & Yes & No & Yes & $\begin{array}{l}\text { determine } \\
\text { Cannot }\end{array}$ & No & No & $\begin{array}{l}\text { determine } \\
\text { Cannot }\end{array}$ & Yes & No & $\begin{array}{l}\text { applicable } \\
\text { Not }\end{array}$ & No & Poor \\
\hline Mehra & ves & No & Cannot & Cannot & No & res & $\begin{array}{l}\text { determine } \\
\text { Cannot }\end{array}$ & yes & No & $\begin{array}{l}\text { determinine } \\
\text { cannot }\end{array}$ & ves & No & $\begin{array}{l}\text { appotitable } \\
\text { Not }\end{array}$ & yes & Fair \\
\hline ru, cai & res & res & $\begin{array}{l}\text { determine } \\
\text { Cannot }\end{array}$ & $\begin{array}{l}\text { determinin } \\
\text { Cannot }\end{array}$ & мо & Cannot & $\begin{array}{l}\text { determine } \\
\text { Cannot }\end{array}$ & No & No & $\begin{array}{l}\text { determmine } \\
\text { Cannot }\end{array}$ & res & No & $\begin{array}{l}\text { applicable } \\
\text { Not }\end{array}$ & No & Poor \\
\hline Zheng, xiong & Yes & No & $\begin{array}{l}\text { determine } \\
\text { Cannot }\end{array}$ & $\begin{array}{l}\text { detetmine } \\
\text { Cannot }\end{array}$ & No & $\begin{array}{l}\text { determine } \\
\text { Cannot }\end{array}$ & $\begin{array}{l}\text { determine } \\
\text { Cannot }\end{array}$ & № & № & $\begin{array}{l}\text { determmine } \\
\text { Cannot }\end{array}$ & res & № & $\begin{array}{l}\text { applicable } \\
\text { Not }\end{array}$ & Yes & Poor \\
\hline Mivara & res & Yes & $\begin{array}{l}\text { deter } \\
\text { yes }\end{array}$ & $\begin{array}{l}\text { determine } \\
\text { Yes }\end{array}$ & No & $\begin{array}{l}\text { determine } \\
\text { Yes }\end{array}$ & $\begin{array}{l}\text { determine } \\
\text { cannot }\end{array}$ & Yes & № & $\begin{array}{l}\text { determine } \\
\text { cannot }\end{array}$ & res & No & $\begin{array}{l}\text { applicable } \\
\text { Not }\end{array}$ & No & air \\
\hline dela pion & veser & res & 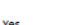 & 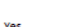 & . & o & determine & . & e. & determine & . & o & applicable & 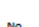 & 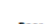 \\
\hline Detionse & sestis & fes & ses & tes & 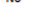 & test & determine & wo & 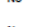 & determine & fes & wo & applicable & 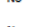 & 年 \\
\hline Yin, Yang & res & res & $\begin{array}{l}\text { Cannot } \\
\text { determine }\end{array}$ & Yes & No & $\begin{array}{l}\text { Canot } \\
\text { determine }\end{array}$ & $\begin{array}{l}\text { Canot } \\
\text { determine }\end{array}$ & No & No & $\begin{array}{l}\text { Cannot } \\
\text { deteremine }\end{array}$ & Yes & No & $\begin{array}{l}\text { Not } \\
\text { appolicable }\end{array}$ & No & Poor \\
\hline Gabbazzi & res & ves & Cannot & Cannot & No & Cannot & cannot & Yes & No & Cannot & Yes & No & Not & Yes & Fair \\
\hline shi, zuo & res & ves & $\begin{array}{l}\text { Cannot } \\
\text { determine }\end{array}$ & Yes & No & $\begin{array}{l}\text { Cannot } \\
\text { determine }\end{array}$ & $\begin{array}{l}\text { Cannot } \\
\text { determine }\end{array}$ & No & No & $\begin{array}{l}\text { Cannot } \\
\text { determine }\end{array}$ & ves & No & $\begin{array}{l}\text { Not } \\
\text { applicable }\end{array}$ & № & Poor \\
\hline
\end{tabular}




\begin{tabular}{|c|c|c|c|c|c|c|c|c|c|c|c|c|c|c|c|}
\hline cho & ves & res & Cannot & ves & No & yes & cannot & res & yes & Cannot & ves & No & Not & ves & Fair \\
\hline Allenbach & res & res & $\begin{array}{l}\text { Cannot } \\
\text { Cannot }\end{array}$ & res & No & № & $\begin{array}{l}\text { determine } \\
\text { cannot }\end{array}$ & No & оㅡㄴ & $\begin{array}{l}\text { Determine } \\
\text { Cannot }\end{array}$ & res & No & $\begin{array}{l}\text { Sappictiole } \\
\text { Not }\end{array}$ & No & poor \\
\hline Robiotti & ves & № & $\begin{array}{l}\text { Des } \\
\text { verm }\end{array}$ & Cannot & No & Cannot & $\begin{array}{l}\text { Determine } \\
\text { cannot }\end{array}$ & yes & yes & $\begin{array}{l}\text { oetermine } \\
\text { Cannot }\end{array}$ & res & No & $\begin{array}{l}\text { Sappictiole } \\
\text { Not }\end{array}$ & No & Poor \\
\hline OpensafEEYY & ves & No & Yes & $\begin{array}{l}\text { Sectimine } \\
\text { Cannot }\end{array}$ & No & 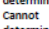 & $\begin{array}{l}\text { cedentint } \\
\text { cannot }\end{array}$ & Yes & № & $\begin{array}{l}\text { Cereninine } \\
\text { Cannot }\end{array}$ & res & Yes & $\begin{array}{l}\text { Sot } \\
\text { Not }\end{array}$ & ves & Fair \\
\hline $\begin{array}{l}\text { Colloborative } \\
\text { Borobia }\end{array}$ & res & res & Yes & $\begin{array}{l}\text { detetrmine } \\
\text { Cannot }\end{array}$ & No & $\begin{array}{l}\text { deter } \\
\text { No }\end{array}$ & $\begin{array}{l}\text { determine } \\
\text { cannot }\end{array}$ & No & No & $\begin{array}{l}\text { detetrmine } \\
\text { Cannot }\end{array}$ & res & No & $\begin{array}{l}\text { appoticable } \\
\text { Not }\end{array}$ & no & Poor \\
\hline Giacomelli & res & ves & Cannot & $\begin{array}{l}\text { leter } \\
\text { yes }\end{array}$ & No & Cannot & $\begin{array}{l}\text { determine } \\
\text { cannot }\end{array}$ & o. & No & $\begin{array}{l}\text { Determine } \\
\text { Cannot }\end{array}$ & res & No & $\begin{array}{l}\text { Sappicteble } \\
\text { Not }\end{array}$ & No & Poor \\
\hline shah & res & ves & $\begin{array}{l}\text { determin } \\
\text { cannot }\end{array}$ & ves & No & $\begin{array}{l}\text { determin } \\
\text { cannot }\end{array}$ & 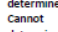 & yes & ves & $\begin{array}{l}\text { Defermine } \\
\text { Cannot }\end{array}$ & res & Yes & $\begin{array}{l}\text { Sappictable } \\
\text { Not }\end{array}$ & No & Fair \\
\hline Bello-chavolla & res & res & $\begin{array}{l}\text { cannot } \\
\text { conte }\end{array}$ & ves & No & $\begin{array}{l}\text { cannot } \\
\text { canot }\end{array}$ & $\begin{array}{l}\text { cetermint } \\
\text { cannot }\end{array}$ & No & мо & $\begin{array}{l}\text { oetermine } \\
\text { cannot } \\
\text { calot }\end{array}$ & res & No & $\begin{array}{l}\text { Sappictere } \\
\text { Not }\end{array}$ & No & poor \\
\hline kolin & res & res & cannot & res & No & 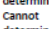 & $\begin{array}{l}\text { Selerminte } \\
\text { cannot. }\end{array}$ & yes & res & $\begin{array}{l}\text { Determine } \\
\text { cannot }\end{array}$ & res & No & $\begin{array}{l}\text { Sot } \\
\text { Noticticie }\end{array}$ & No & Fair \\
\hline Lubetziky & res & ves & $\begin{array}{l}\text { Determine } \\
\text { Cannot }\end{array}$ & yes & No & $\begin{array}{l}\text { determin } \\
\text { Cannot }\end{array}$ & $\begin{array}{l}\text { determine } \\
\text { cannot }\end{array}$ & № & мо & $\begin{array}{l}\text { determine } \\
\text { cannot }\end{array}$ & res & No & $\begin{array}{l}\text { alppoticable } \\
\text { Not }\end{array}$ & No & Poor \\
\hline Goval & res & Yes & $\begin{array}{l}\text { deter } \\
\text { Yes }\end{array}$ & Cannot & No & $\begin{array}{l}\text { determina } \\
\text { Cannot }\end{array}$ & $\begin{array}{l}\text { determine } \\
\text { cannot }\end{array}$ & No & No & $\begin{array}{l}\text { oetermine } \\
\text { Cannot }\end{array}$ & ves & No & $\begin{array}{l}\text { Sappictable } \\
\text { Not }\end{array}$ & No & Poor \\
\hline Feng & res & res & ves & $\begin{array}{l}\text { Cannot } \\
\text { Content }\end{array}$ & No & $\begin{array}{l}\text { Cannot } \\
\text { Contents }\end{array}$ & $\begin{array}{l}\text { cannot } \\
\text { can }\end{array}$ & yes & No & $\begin{array}{l}\text { determine } \\
\text { cannot }\end{array}$ & yes & No & $\begin{array}{l}\text { Sappictable } \\
\text { Not }\end{array}$ & No & poor \\
\hline yao & res & ves & ves & Cannot & No & $\begin{array}{l}\text { Determini } \\
\text { Cannot }\end{array}$ & $\begin{array}{l}\text { determine } \\
\text { cannot }\end{array}$ & мо & No & $\begin{array}{l}\text { oetermine } \\
\text { Cannot }\end{array}$ & Yes & No & $\begin{array}{l}\text { Sotplicale } \\
\text { Not }\end{array}$ & no & Poor \\
\hline Sami & res & res & Cannot & $\begin{array}{l}\text { detetrmine } \\
\text { cannot }\end{array}$ & No & $\begin{array}{l}\text { determin } \\
\text { Cannot }\end{array}$ & $\begin{array}{l}\text { determine } \\
\text { Cannot }\end{array}$ & No & No & $\begin{array}{l}\text { determine } \\
\text { Cannot }\end{array}$ & yes & No & $\begin{array}{l}\text { alapticable } \\
\text { Not }\end{array}$ & No & Poor \\
\hline Almazeedi & res & res & $\begin{array}{l}\text { detert } \\
\text { ves }\end{array}$ & $\begin{array}{l}\text { Determ } \\
\text { Yes }\end{array}$ & No & $\begin{array}{l}\text { determin } \\
\text { Cannot }\end{array}$ & $\begin{array}{l}\text { deterrmine } \\
\text { Cannot }\end{array}$ & № & No & $\begin{array}{l}\text { determinie } \\
\text { Cannot }\end{array}$ & Yes & No & $\begin{array}{l}\text { applicable } \\
\text { Not }\end{array}$ & Yes & Poor \\
\hline carillo-vega & res & Yes & Yes & Cannot & No & $\begin{array}{l}\text { determini } \\
\text { Cannot }\end{array}$ & $\begin{array}{l}\text { determine } \\
\text { Cannot }\end{array}$ & No & No & $\begin{array}{l}\text { oetermine } \\
\text { Cannot }\end{array}$ & ves & No & Not & ves & Poor \\
\hline Vanover & res & yes & yes & $\begin{array}{l}\text { yes } \\
\text { Yon }\end{array}$ & No & cannot & $\begin{array}{l}\text { ceremoti } \\
\text { cannot }\end{array}$ & yes & No & $\begin{array}{l}\text { cannot } \\
\text { const }\end{array}$ & yes & No & Not & yes & Fair \\
\hline Hamer & res & No & Cannot & res & No & Cannot & cannot & yes & No & $\begin{array}{l}\text { oetermine } \\
\text { Cannot }\end{array}$ & No & No & $\begin{array}{l}\text { Sotplictile } \\
\text { Not }\end{array}$ & No & Fair \\
\hline Regina & res & Yes & $\begin{array}{c}\text { oetermine } \\
\text { Cannot }\end{array}$ & Yes & No & $\begin{array}{l}\text { determini } \\
\text { cannot }\end{array}$ & $\begin{array}{l}\text { determinne } \\
\text { Cannot }\end{array}$ & No & No & $\begin{array}{l}\text { detetrmine } \\
\text { Cannot }\end{array}$ & yes & No & $\begin{array}{l}\text { alppictable } \\
\text { Not }\end{array}$ & yes & Poor \\
\hline ISARIC & No & No & $\begin{array}{l}\text { determine } \\
\text { cannot }\end{array}$ & cannot & No & $\begin{array}{l}\text { determin } \\
\text { cannot } \\
\text { d }\end{array}$ & $\begin{array}{l}\text { deterninie } \\
\text { cannot }\end{array}$ & No & No & $\begin{array}{l}\text { determine } \\
\text { cannot }\end{array}$ & yes & No & $\begin{array}{l}\text { applicable } \\
\text { Not }\end{array}$ & No & Poor \\
\hline
\end{tabular}

\section{Discussion}

This rapid review of 67 observational studies found substantial uncertainty arising from the recording of smoking status. Notwithstanding recording uncertainties, compared with national prevalence estimates, recorded current and former smoking rates in most studies were lower than expected. From available data, there was insufficient evidence to establish whether current and/or former smoking status is associated with SARS-CoV-2 infection, hospitalisation or mortality. There was limited evidence from 'fair' quality studies that disease severity in those hospitalised for COVID-19 is greater in current but not former smokers compared with never smokers. There were inconsistent results on the association of current or past compared with never smoking and increased mortality from COVID-19.

\section{Infection by smoking status}

There is currently no evidence that current or former smokers in the community are more likely to test positive compared with never smokers. Infection positivity rates estimated among random samples will be more informative than currently available data. Smoking status is being collected in at least one large representative infection and antibody survey in the UK ${ }^{89}$.

\section{Hospitalisation and disease severity by smoking status}

As reported elsewhere, smoking prevalence among multiple hospital cohorts was consistently lower than national estimates 16 . In contrast, there was no evidence that current or former smokers are at lower risk of hospitalisation for COVID-19 compared 
with never smokers among those identified as testing positive in the community. There was some limited evidence that current smokers are at increased risk of greater disease severity compared with never smokers.

\section{Mortality by smoking status}

In three 'fair' quality studies, there was inconsistent evidence on the association of smoking status and the risk of death from COVID-19. It should be noted that these early studies did not follow all patients for a sufficient period of time to report mortality outcomes.

\section{Issues complicating interpretation}

Interpretation of these early studies is complicated by several factors (see Figure 9). First, exposure to SARS-CoV-2 is heterogeneous with different subgroups being at heightened risk of infection at different stages of the pandemic. This will likely introduce bias in studies assessing the rate of infection by smoking status conducted early on in the pandemic. Second, current smokers may be more likely to meet local criteria for community testing due to increased prevalence of symptoms consistent with SARS-CoV2 infection, such as cough, increased sputum production or altered sense of smell or taste. Third, testing for acute infection requires swabbing of the mucosal epithelium, which may be disrupted in current smokers, thus altering the sensitivity of the assay.

Fourth, most included studies relied on electronic health records (EHRs) as the source of information on smoking status. Research shows large discrepancies between EHRs and actual behaviour ${ }^{90}$. Known failings of EHRs include implausible longitudinal changes, such as former smokers being recorded as never smokers at subsequent hospital visits ${ }^{90}$. Misreporting on the part of the patient (perhaps due to perceived stigmatisation) has also been observed, with biochemical measures showing higher rates of smoking behaviour compared with self-report in hospitalised patients in the US ${ }^{91}$. It is hence possible that under-reporting of current and former smoking status occurred across the included studies. Fifth, individuals with severe COVID-19 symptoms may have stopped smoking prior to admission to a care facility and may therefore not have been recorded as current smokers (i.e. reverse causality).

Sixth, smokers with COVID-19 may be less likely to receive a SARS-CoV-2 test or present to hospital due to lack of access to healthcare and may be more likely to die in the community from sudden complications (i.e. self-selection). Seventh, if there is a protective effect of nicotine on COVID-19 disease outcomes, abrupt nicotine withdrawal 
upon hospitalisation may lead to worse outcomes ${ }^{12}$. Eight, during periods of heightened demand of limited healthcare resources, current smokers with extensive comorbidities may have reduced priority for intensive care admission, thus leading to higher in-hospital mortality.

Another important issue is that the reason for hospitalisation varies by country and time in the epidemic. For example, initial cases may have been hospitalised for isolation and quarantine reasons and not due to medical necessity. It is plausible that this may have skewed early data towards less severe cases. In addition, the observed association between current smoking and disease severity may be explained by collider bias, where conditioning on a collider (e.g. testing or hospitalisation) by design or analysis may introduce a spurious association between smoking (a potential cause of testing or hospitalisation) and SARS-CoV-2 infection/adverse outcomes from COVID-19 (potentially exacerbated by smoking $)^{92}$.

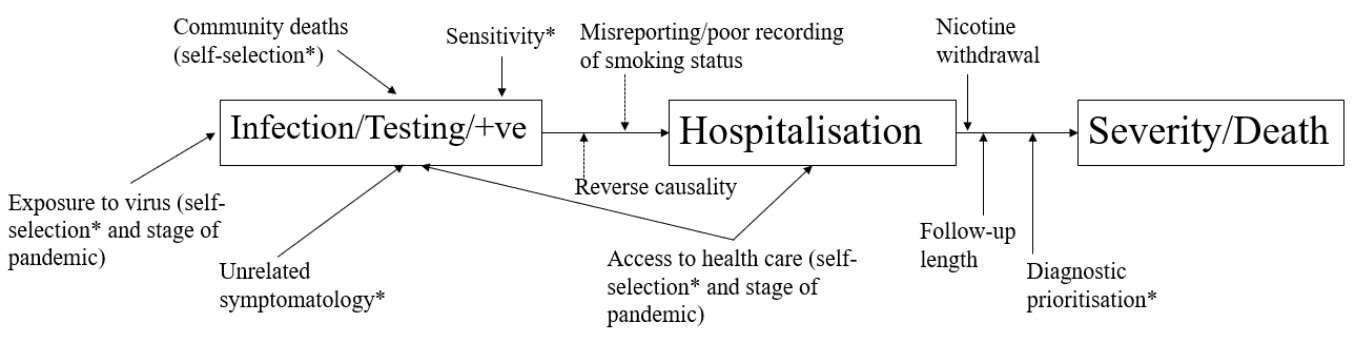

Figure 9. A schematic of some interpretation issues for the association of smoking and SARS-CoV2/COVID-19. * indicates potential confounding with smoking status

\section{Limitations}

This rapid review was limited by not having two independent reviewers extracting data, limiting the search to one electronic database and one pre-print server and by not including at least two large population surveys due to their reliance on self-reported SARS-CoV-2 infection (which means they are not currently meeting our eligibility criteria $^{93,94}$. Population surveys - particularly with linked health data - will be included in future review versions to help mitigate some of the limitations of healthcare based observational studies.

\section{Implications for research, policy and practice}

Further scientific research is needed to resolve the mixed findings summarised in our review. First, clinical trials of the posited therapeutic effect of nicotine could have important implications both for smokers and for improved understanding of the SARS- 
CoV-2 virus. Such trials should focus on medicinal nicotine (as smoked tobacco is a dirty delivery mechanism that could mask beneficial effects) and potentially differentiate between different modes of delivery (i.e. inhaled vs. not) since this can affect pharmacokinetics ${ }^{95}$ (and thus potential therapeutic effects). A second research priority would be a large, representative (randomly sampled) population survey with a validated assessment of smoking status which distinguishes between recent and long-term exsmokers - ideally biochemically verified - and assesses seroprevalence and links to health records. In the meantime, public-facing messages about the possible protective effect of smoking or nicotine are premature. In our view, until there is further research, the quality of the evidence does not justify the huge risk associated with a message likely to reach millions of people that a lethal activity, such as smoking, may protect against COVID-19. It continues to be appropriate to recommend smoking cessation and emphasise the role of alternative nicotine to support smokers to stop as part of public health efforts during COVID-19. At the very least, smoking cessation reduces acute risks from cardiovascular disease and could reduce demands on the healthcare system ${ }^{96}$. GPs and other healthcare providers can play a crucial role - brief, high-quality and free online training is available from the National Centre for Smoking Cessation and Training

\section{Conclusion}

Across 67 observational studies, there is substantial uncertainty arising from the recording of smoking status on whether current and/or former smoking status is associated with SARS-CoV-2 infection, hospitalisation or mortality. There is limited evidence that current smoking compared with never is associated with greater disease severity in those hospitalised for COVID-19.

\section{Acknowledgements}

An original short review for the Royal College of Physicians was converted to an extended living review after a request by Martin Dockrell, Tobacco Control Lead, Public Health England. All scientific decisions were made by the authors independently of funders and external organisations. The authors would also like to thank Rosemary Koper for her assistance in running the electronic searches.

\section{References}

1 Guan W, Ni Z, Hu YY, et al. Clinical Characteristics of Coronavirus Disease 2019 in China. N EnglJ Med 2020; : NEJMoa2002032.

2 Hoffmann M, Kleine-Weber H, Schroeder S, et al. SARS-CoV-2 Cell Entry Depends 
on ACE2 and TMPRSS2 and Is Blocked by a Clinically Proven Protease Inhibitor. Cell 2020; published online March 5. DOI:10.1016/j.cell.2020.02.052.

3 Brake SJ, Barnsley K, Lu W, McAlinden KD, Eapen MS, Sohal SS. Smoking Upregulates Ang iotensin-Converting Enzyme-2 Receptor: A Potential Adhesion Site for Novel Coronavirus SARS-CoV-2 (Covid-19). J Clin Med 2020, Vol 9, Page 841 2020; 9: 841. $4 \quad$ Cai G. Bulk and Single-Cell Transcriptomics Identify Tobacco-Use Disparity in Lung Gene Expression of ACE2, the Receptor of 2019-nCov. 2020; published online March 2. DOI:10.20944/PREPRINTS202002.0051.V3.

5 Oakes JM, Fuchs RM, Gardner JD, Lazartigues E, Yue X. Nicotine and the reninangiotensin system. Am. J. Physiol. - Regul. Integr. Comp. Physiol. 2018; 315: R895-906. 6 Denholm JT, Gordon CL, Johnson PD, et al. Hospitalised adult patients with pandemic (H1N1) 2009 influenza in Melbourne, Australia. Med J Aust 2010; 192: 84-6. 7 Abadom TR, Smith AD, Tempia S, Madhi SA, Cohen C, Cohen AL. Risk factors associated with hospitalisation for influenza-associated severe acute respiratory illness in South Africa: A case-population study. Vaccine 2016; 34: 5649-55.

8 Almirall J, González CA, Balanzó X, Bolíbar I. Proportion of community-acquired pneumonia cases attributable to tobacco smoking. Chest 1999; 116: 375-9.

9 Feldman C, Anderson R. Cigarette smoking and mechanisms of susceptibility to infections of the respiratory tract and other org an systems. J. Infect. 2013; 67: 169-84. 10 Dye JA, Adler KB. Occasional review Effects of cigarette smoke on epithelial cells of the respiratory tract. Thorax 1994; 49: 825-34.

11 Vardavas Cl, Nikitara K. COVID-19 and smoking: A systematic review of the evidence. Tob Induc Dis 2020; 18: 20.

12 Farsalinos K, Niaura R, Le Houezec J, et al. Editorial: Nicotine and SARS-CoV-2: COVID-19 may be a disease of the nicotinic cholinergic system. Toxicol Reports 2020; published online April. DOI:10.1016/j.toxrep.2020.04.012.

13 Emami A, Javanmardi F, Pirbonyeh N, Akbari A. Prevalence of Underlying Diseases in Hospitalized Patients with COVID-19: a Systematic Review and Meta-Analysis. Arch Acad Emerg Med 2020; 8: e35.

14 Arabia S, Health D, Arabia S, et al. Prevalence, Severity and Mortality associated with COPD and Smoking in patients with COVID-19: A Rapid Systematic Review and MetaAnalysis. medRxiv 2020; : 2020.03.25.20043745.

15 Patanavanich R, Glantz SA. Smoking is Associated with COVID-19 Progression: A Meta-Analysis. medRxiv 2020. DOI:10.14171/j.2095-5944.sg.2014.02.004.

16 Farsalinos K, Barbouni A, Niaura R. Smoking, vaping and hospitalization for COVID-19. Qeios 2020; published online March 25. DOI:10.32388/Z6908A.8. 
Res DOI:10.1093/NTR/NTAA059.

18 Tricco AC, Antony J, Zarin W, et al. A scoping review of rapid review methods. BMC Med 2015; 13: 224.

19 National Heart Lung and Blood Institute. Study Quality Assessment Tools. Natonal Institutes Heal. 2018; : 1-35.

20 R Core Team. The R Project for Statistical Computing. 2013; : 1-12.

$21 \quad$ Higgins JPT, Wells GA. Cochrane handbook for systematic reviews of interventions. 2011.

22 Higgins JPT, Thompson SG, Deeks لע, Altman DG. Measuring inconsistency in meta-analyses. Br. Med. J. 2003; 327: 557-60.

23 Guan W, Liang W, Zhao Y, et al. Comorbidity and its impact on 1590 patients with Covid-19 in China: A Nationwide Analysis. Eur Respir J 2020; : 2000547.

24 Lian J, Jin X, Hao S, et al. Analysis of Epidemiological and Clinical Features in Older Patients With Coronavirus Disease 2019 (COVID-19) Outside Wuhan. Clin Infect Dis 2020; 2019: 1-8.

25 Jin X, Lian JS, Hu JH, et al. Epidemiological, clinical and virological characteristics of 74 cases of coronavirus-infected disease 2019 (COVID-19) with gastrointestinal symptoms. Gut 2020; published online March 24. DOI:10.1136/gutjnl-2020-320926. 26 Chen T, Wu D, Chen H, et al. Clinical characteristics of 113 deceased patients with coronavirus disease 2019: Retrospective study. BMJ 2020; 368. DOI:10.1136/bmj.m1091. 27 Zhou F, Yu T, Du R, et al. Clinical course and risk factors for mortality of adult inpatients with COVID-19 in Wuhan, China: a retrospective cohort study. Lancet 2020; 0. DOI:10.1016/s0140-6736(20)30566-3.

28 Mo $P$, Xing $Y$, Xiao $Y$, et al. Clinical characteristics of refractory COVID-19 pneumonia in Wuhan, China. Clin Infect Dis 2020; published online March 16. DOI:10.1093/cid/ciaa270.

29 Zhang J, Dong X, Cao Y, et al. Clinical characteristics of 140 patients infected with SARS-CoV-2 in Wuhan, China. Allergy 2020; : all.14238.

30 Wan S, Xiang Y, Fang W, et al. Clinical features and treatment of COVID-19 patients in northeast Chongqing. J Med Virol 2020; : 1-10.

31 Liu W, T ao Z-W, Wang L, et al. Analysis of factors associated with disease outcomes in hospitalized patients with 2019 novel coronavirus disease. Chin Med J (Engl) 2020; $133: 1$.

32 Huang C, Wang Y, LiX, et al. Clinical features of patients infected with 2019 novel coronavirus in Wuhan, China. Lancet 2020; 395: 497-506.

33 Zhang $\mathrm{X}, \mathrm{Cai} \mathrm{H}, \mathrm{Hu}$ J, et al. Epidemiological, clinical characteristics of cases of SARS-CoV-2 infection with abnormal imaging findings. Int J Infect Dis 2020; 94: 81-7. 
34 Guo T, Fan Y, Chen M, et al. Cardiovascular Implications of Fatal Outcomes of Patients With Coronavirus Disease 2019 (COVID-19). JAMA Cardiol 2020; 2019. DOI:10.1001/jamacardio.2020.1017.

35 Liu R, Ming X, Zhu H, et al. Association of Cardiovascular Manifestations with Inhospital Outcomes in Patients with COVID-19: A Hospital Staff Data. medRxiv 2020; : 2020.02.29.20029348.

36 Huang $Y$, Yang $R, X u Y$, et al. Clinical characteristics of 36 non-survivors with COVID-19 in Wuhan, China. medRxiv 2020; : 2020.02.27.20029009.

$37 \mathrm{Xu} \mathrm{HH}, \mathrm{Hou} \mathrm{K}, \mathrm{Xu} \mathrm{HH}$, et al. Acute Myocardial Injury of Patients with Coronavirus Disease 2019. medRxiv 2020; : 2020.03.05.20031591.

38 Li J, Li S, Cai Y, et al. Epidemiological and Clinical Characteristics of 17 Hospitalized Patients with 2019 Novel Coronavirus Infections Outside Wuhan, China. medRxiv 2020; : 2020.02.11.20022053.

$39 \mathrm{Hu}$ L, Chen S, Fu Y, et al. Risk Factors Associated with Clinical Outcomes in 323

COVID-19 Patients in Wuhan, China. medRxiv 2020; : 2020.03.25.20037721.

40 Wang R, Pan M, Zhang X, et al. Epidemiological and clinical features of 125 Hospitalized Patients with COVID-19 in Fuyang, Anhui, China. Int J Infect Dis 2020; : 127065.

41 Dong X, Cao Y, Lu X, et al. Eleven Faces of Coronavirus Disease 2019. Allergy 2020; : 1-11.

42 Shi Y, YuX, Zhao H, Wang H, Zhao R, Sheng J. Host susceptibility to severe COVID-19 and establishment of a host risk score: Findings of 487 cases outside Wuhan. Crit Care 2020; 24: 2-5.

43 Yang $X, Y u$ Y, Xu J, et al. Clinical course and outcomes of critically ill patients with SARS-CoV-2 pneumonia in Wuhan, China: a single-centered, retrospective, observational study. Lancet Respir Med 2020; 2600: 1-7.

44 Zheng KI, Gao F, Wang X-B, et al. Obesity as a risk factor for greater severity of COVID-19 in patients with metabolic associated fatty liver disease. Metabolism 2020; : 154244.

45 Liao Y, Feng Y, Wang B, et al. Clinical Characteristics and Risk factors for developed COVID-19 patients transferring to designated hospital from Jianghan Fang cang shelter Hospital: a retrospective, Summary: 2020; :1-16.

46 Shi P, Ren G, Yang J, et al. Clinical characteristics of imported and secondgeneration COVID-19 cases outside Wuhan, China: A multicenter retrospective study. 2020. DOI:10.1101/2020.04.19.20071472.

47 Yu T, Cai S, Zheng Z, et al. Association between clinical manifestations and prognosis in patients with COVID-19. Clin Ther 2020; xxx: 1-9. 
48 Zheng Y, Xiong C, Liu Y, et al. Epidemiological and Clinical Characteristics Analysis of COVID-19 in the Surrounding Areas of Wuhan, Hubei Province in 2020. Pharmacol Res 2020; 157: 104821.

49 Yin R, Yang Z, Wei Y, et al. Clinical characteristics of 106 patients with neurological diseases and co-morbid coronavirus disease 2019: a retrospective study. medRxiv 2020; : 2020.04.29.20085415.

50 Feng Y, Ling Y, Bai T, et al. COVID-19 with Different Severity: A Multi-center Study of Clinical Features. Am J Respir Crit Care Med 2020; : 1-53.

51 Yao Q, Wang P, Wang X, et al. Retrospective study of risk factors for severe SARS-Cov-2 infections in hospitalized adult patients. Polish Arch Intern Med 2020. DOI:10.20452/pamw.15312.

52 Rentsch CT, Kidwai-Khan F, T ate JP, et al. Covid-19 T esting, Hospital Admission, and Intensive Care Among 2,026,227 United States Veterans Aged 54-75 Years. medRxiv 2020; : 2020.04.09.20059964.

53 Petrilli CM, Jones SA, Yang J, et al. Factors associated with hospitalization and critical illness among 4,103 patients with COVID-19 disease in New York City. medRxiv 2020; : 2020.04.08.20057794.

54 Chow N, Fleming-Dutra K, Gierke R, et al. Preliminary Estimates of the Prevalence of Selected Underlying Health Conditions Among Patients with Coronavirus Disease 2019 - United States, February 12-March 28, 2020. Morb Mortal Wkly Rep 2020; 69: 382-6. 55 Argenziano MG, Bruce SL, Slater CL, et al. Characterization and Clinical Course of 1000 Patients with COVID-19 in New York: retrospective case series. medRxiv 2020; : 2020.04.20.20072116.

56 Richardson S, Hirsch JS, Narasimhan M, et al. Presenting Characteristics, Comorbidities, and Outcomes Among 5700 Patients Hospitalized With COVID-19 in the New York City Area. JAMA 2020; 10022: 1-8.

57 Magagnoli J, Narendran S, Pereira F, et al. Outcomes of hydroxychloroquine usage in United States veterans hospitalized with Covid-19. medRxiv 2020; : 2020.04.16.20065920.

58 Gold JAW, Wong KK, Szablewski CM, Patel PR, Rossow J, Silva J. Characteristics and Clinical Outcomes of Adult Patients Hospitalized with COVID-19 - Georgia, March 2020. 2020; 69. https://www.cdc.gov/mmwr/volumes/69/wr/mm6918e1. htm? S_cid=mm6918e1_w.

59 Shi H, Zuo Y, Yalavarthi S, et al. Neutrophil calprotectin identifies severe pulmonary disease in COVID-19 Hui. medRxiv 2020; : 1-15.

60 Robilotti E V, Babady NE, Ph D, et al. Determinants of Severity in Cancer Patients with COVID-19 Illness. medRxiv 2020; : 1-19. 
Shah SJ, Barish PN, Prasad PA, et al. Clinical features, diagnostics, and outcomes of patients presenting with acute respiratory illness: a comparison of patients with and without COVID-19. medRxiv 2020; : 2020.05.02.20082461.

62 Lubetzky M, Aull M, Craig-Shapiro R, et al. Kidney Allograft Recipients Diagnosed with Coronavirus Disease-2019: A Single Center Report. medRxiv 2020; : 2020.04.30.20086462.

63 Goyal P, Choi لע, Pinheiro LC, et al. Clinical Characteristics of Covid-19 in New York City. N EngIJ Med 2020; published online April 17. DOI:10.1056/nejmc2010419.

64 Niedzweidz C, O'Donnell CA, Jani BD, et al. Ethnic and socioeconomic differences in SARS-CoV-2 infection: prospective cohort study using UK Biobank. 2020.

DOI:10.1101/2020.04.22.20075663.

65 Cho ER, Jha P. Smoking and the risk of COVID-19 infection in the UK Biobank Prospective Study. 2020; : 10-3.

66 Collaborative TO, Williamson E, Walker AJ, et al. OpenSAFELY: factors associated with COVID-19-related hospital death in the linked electronic health records of 17 million adult NHS patients. medRxiv 2020; : 2020.05.06.20092999.

67 Kolin DA, Kulm S, Elemento O. Clinical and Genetic Characteristics of Covid-19 Patients from UK Biobank. medRxiv 2020; : 2020.05.05.20075507.

68 Hamer M, Kivimäki M, Gale CR, Batty GD. Lifestyle Risk Factors for Cardiovascular Disease in Relation to COVID-19 Hospitalization: A Community-Based Cohort Study of 387, 109 Adults in UK Division of Surgery and Interventional Sciences, Faculty Medical Sciences, University College London, L. 2020; : 1-11.

69 de Lusignan S, Dorward J, Correa A, et al. Risk factors for SARS-CoV-2 among patients in the Oxford Royal College of General Practitioners Research and Surveillance Centre primary care network: a cross-sectional study. Lancet Infect Dis 2020; 0. DOI:10.1016/S1473-3099(20)30371-6.

70 Miyara M, T ubach F, POURCHER V, et al. Low incidence of daily active tobacco smoking in patients with symptomatic COVID-19. Qeios 2020; published online April 21. DOI:10.32388/WPP19W.3.

71 Fontanet A, Tondeur L, Madec Y, et al. Cluster of COVID-19 in northern France: A retrospective closed cohort study. medRxiv 2020; : 2020.04.18.20071134.

72 Hadjadj J, Yatim N, Barnabei L, et al. Impaired type I interferon activity and exacerbated inflammatory responses in severe Covid-19 patients. medRxiv 2020; : 2020.04.19.20068015.

73 Allenbach Y, Saadoun D, Maalouf G, et al. Multivariable prediction model of intensive care unit transfer and death: a French prospective cohort study of COVID-19 patients. medRxiv 2020; : 2020.05.04.20090118. 
74 Solis P, Carreno H. COVID-19 Fatality and Comorbidity Risk Factors among Diagnosed Patients in Mexico. 2020. DOI:10.1101/2020.04.21.20074591.

75 Bello-Chavolla OY, Bahena-Lopez JP, Antonio-Villa NE, et al. Predicting mortality attributable to SARS-CoV-2: A mechanistic score relating obesity and diabetes to COVID19 outcomes in Mexico. medRxiv 2020; 52: 2020.04.20.20072223.

76 Carrillo-Vega MF, Salinas-Escudero G, Garcia-Peña C, Gutierrez-Robledo LM, Parra-Rodriguez L. Early estimation of the risk factors for hospitalisation and mortality by COVID-19 in Mexico. medRxiv 2020; : 2020.05.11.20098145.

77 Rodriguez-Cola M, Jimenez-Velasco I, Gutierrez-Henares F, et al. Clinical features of coronavirus disease 2019 (COVID-19) in a cohort of patients with disability due to spinal cord injury. 2020. DOI:10.1101/2020.04.20.20072918.

78 Rica R de la, Borges M, Aranda M, et al. Low albumin levels are associated with poorer outcomes in a case series of COVID-19 patients in Spain: a retrospective cohort study. medRxiv 2020; : 2020.05.07.20094987.

79 Borobia AM, Carcas AJ, Arnalich F, Alvarez-Sala R, Montserrat J, Quintana M. A cohort of patients with COVID-19 in a major teaching hospital in Europe. medRxiv 2020. DOI:10.1101/2020.04.29.20080853.

80 Mehra MR, Desai SS, Kuy S, Henry TD, Patel AN. Cardiovascular Disease, Drug Therapy, and Mortality in Covid-19. N EngIJ Med 2020; : NEJMoa2007621.

81 ISARIC. International Severe Acute Respiratory and Emerging Infection Consortium. 2020. https://isaric.tghn.org/about/.

82 Gaibazzi N, Tuttolomondo D, Guidorossi A, et al. Smoking Prevalence is Low in Symptomatic Patients Admitted for COVID-19. medRxiv 2020; : 2020.05.05.20092015. 83 Giacomelli A, Ridolfo AL, Milazzo L, et al. 30-day mortality in patients hospitalized with COVID-19 during the first wave of the Italian epidemic: a prospective cohort study. medRxiv 2020; : 2020.05.02.20088336.

84 Sami R, Soltaninejad F, Amra B, et al. A one-year hospital-based prospective CVOID-19 open-cohort in the Eastern Mediterranean region: The Khorshid COVID Cohort (KCC) study. medRxiv 2020; : 2020.05.11.20096727.

85 Yanover AC, Mizrahi B, Kalkstein N, Marcus K, Akiva P, Barer Y. What factors increase the risk of complications in SARS-CoV-2 positive patients ? A cohort study in a nationwide Israeli health organization. 2020.

86 Kim ES, Chin BS, Kang CK, et al. Clinical Course and Outcomes of Patients with Severe Acute Respiratory Syndrome Coronavirus 2 Infection: a Preliminary Report of the First 28 Patients from the Korean Cohort Study on COVID-19. J Korean Med Sci 2020; 35: e142.

87 Almazeedi S, Youha S Al, Jamal MH, et al. Clinical Characteristics, Risk Factors and 
Outcomes Among the First Consecutive 1,096 Patients Diagnosed with COVID-19: The Kuwait Experience. medRxiv 2020; : 2020.05.09.20096495.

88 Regina J, Papadimitriou-Olivg eris M, Burger R, et al. Epidemiology, risk factors and clinical course of SARS-CoV-2 infected patients in a Swiss university hospital: an observational retrospective study. medRxiv 2020; : 2020.05.11.20097741.

89 Major home testing prog ramme for coronavirus will track levels of infection in the community - GOV.UK. https://www.gov.uk/government/news/major-home-testingprogramme-for-coronavirus-will-track-levels-of-infection-in-the-community (accessed May 22, 2020).

90 Polubriaginof F, Salmasian H, Albert DA, Vawdrey DK. Challenges with Collecting Smoking Status in Electronic Health Records. AMIA . Annu Symp proceedings AMIA Symp 2017; 2017: 1392-400.

91 Benowitz NL, Schultz KE, Haller CA, Wu AHB, Dains KM, Jacob P. Prevalence of smoking assessed biochemically in an urban public hospital: a rationale for routine cotinine screening. Am J Epidemiol 2009; 170: 885-91.

92 Murray E. Causation in smoking and COVID-19. T witter. 2020. https://twitter.com/EpiEllie/status/1258607277357006849?s=20.

93 Bowyer RCE, Varsavsky T, Carole H. Geo-social gradients in predicted COVID-19 prevalence and severity in Great Britain: results from Affiliations : Corresponding authors: Understanding the geographical distribution of COVID-19 through the general population is key to the provision of ade. 2020.

94 Jackson SE, Brown J, Shahab L, Steptoe A, Fancourt D. COVID-19, smoking, and inequalities: a cross-sectional survey of adults in the UK. Submitted 2020.

95 Shahab L, Brose LS, West R. Novel delivery systems for nicotine replacement therapy as an aid to smoking cessation and for harm reduction: Rationale, and evidence for advantages over existing systems. CNS Drugs 2013; 27: 1007-19.

96 Stead LF, Buitrago D, Preciado N, Sanchez G, Hartmann-Boyce J, Lancaster T. Physician advice for smoking cessation. Cochrane Database Syst. Rev. 2013; 2017. DOI:10.1002/14651858.CD000165.pub4. 